\title{
Flexible substrate sensors for multiplex biomarker monitoring
}

\author{
Desmond Brennan and Paul Galvin, Life Science Interface Group, Tyndall National Institute, University College, Cork, Ireland \\ Address all correspondence to Desmond Brennan at des.brennan@tyndall.ie
}

(Received 23 April 2018; accepted 6 July 2018)

\begin{abstract}
Wearable healthcare technologies should be non-invasive, robust to daily activity/environments, easy to use, and comfortable to wear. Flexible substrate devices for biomarker monitoring can contribute to wearable diagnostic applications. Single-target biosensors have extensively been developed for health-monitoring applications; however, recently multiplex biomarker tests have generated clinical interest. Targeting multiple biomarkers in diagnostic systems (wearable or point of care) offers more focused diagnosis and treatment as changes in a single biomarker can be caused by a series of physiologic conditions. This review highlights flexible substrates that have been successfully demonstrated for multiplex biomarker detection with potential for healthcare monitoring.
\end{abstract}

\section{Introduction}

The single-target biosensor evolution from laboratory to wearable functionality was demonstrated by the classical glucose oxidase (GOx) biosensor, first described by Clarke in 1962 and developed by Medtronic in 2005 for continuous blood glucose monitoring. Single-target sensors have extensively been researched and commercialized for metabolites, antibodies, and proteins, etc. Bio-fluids such as saliva, tear, sweat, and interstitial fluid generate significant research around disease-specific biomarkers. Such fluids are naturally secreted by the body and are painlessly sampled unlike invasive blood draw. Saliva incorporates protein biomarkers ${ }^{[1]}$ relevant to local cell activity and biomolecular function. It is used to monitor creatine, fibrinogen, hemoglobin, triglyceride, glucose levels, and correlates to blood pressure. ${ }^{[2]}$ Tear fluid contains lipids, electrolytes, metabolites, and proteins ${ }^{[3]}$ suitable for disease monitoring. Sweat is extensively used to measure physiologic parameters ${ }^{[4-6]}$ and incorporates protein biomarkers associated with genetic diseases. ${ }^{[7]}$ While single-target assays monitor specific conditions such as diabetes, multiplex protein screening offers improved diagnosis, prognosis, and treatment for cancer. Recent reviews ${ }^{[8-10]}$ have highlighted wearable technology progress around materials, assays, and instrumentation. Flexible substrates offer mechanical properties suitable for wearable devices with Young's moduli compatible with skin applications. ${ }^{[11]}$ Device structure typically consists of flexible layers, including support substrate, active layer, and electrical connections. Careful design ${ }^{[12]}$ avoids device failure (cracking and delamination), caused by stretching and bending. Flexible substrate materials include paper, polymer, and textiles. All offer biocompatibility and robustness during device fabrication and biomolecule immobilization. Natural polymers include cellulose, silk, wool, and cotton, while synthetic polymers include nylon, polyethylene, polyester, and teflon. Wearable biosensors progressed as conductive polymers [polypyrrole, polyaniline (PANI), polythiophene] improved device integration with flexible substrates, delivering good electrical properties for sensor applications. Incorporating graphene, ${ }^{[13-15]}$ carbon nanotubes, ${ }^{[16,17]}$ metal nanoparticles, ${ }^{[18,19]}$ and semiconductor materials into active layers improved electrical and mechanical properties $^{[20]}$ of flexible devices. Issues around fouling and biomolecule interference have been alleviated with biomoleculeselective membranes, immobilization matrices, and antifouling layers. ${ }^{[21-23]}$ Such technologies have contributed to advancement in wearable sensors. Electrochemical sensors offer distinct advantages over optical detection in conformal substrates for biomarker detection. For multiplex assays, multiple fluorophores or microarray approaches require complex optics (e.g., sources, lens, filters, sensor arrays) to be integrated on a single rigid substrate to maintain optical alignment. Flexible electrode systems facilitate bending and twisting having minimal signal impact. Microelectrode arrays manufactured on single substrates facilitate high-density multiplex detection. Signal-to-noise enhancement in optical systems often require long optical pathlengths (e.g., absorption) or high-power sources (e.g., fluorescence) to reach clinically relevant biomarker levels. Such approaches can increase system size, power, and may require specific thermal management. With electrochemical sensors, performance-enhancing approaches include target-selective membranes, materials (e.g., carbon nanotubes) enhancing electrical performance which can be incorporated without size or power impact. In this review, we 
highlight the applications of flexible substrates to multiplex biomarker monitoring with potential for health-monitoring applications.

Saliva, sweat, and tear liquids contain biomarkers (Fig. 1), which are easily accessed using wearable sensor technology, to diagnose and manage a range of clinical conditions. Significant potential for multiplex monitoring exists, due to biomarker diversity within each sample type. In this review, we highlight the applications of flexible substrates to multiplex biomarker monitoring with potential for health-monitoring applications. The potential for wearable biomarker devices to non-invasively monitor a range of physiologic conditions has generated a significant interest as outlined in Table 1, where clinically relevant biomarker ranges to monitor healthcare conditions are highlighted. For each flexible substrate type, device fabrication, assay implementation, and performance is outlined for a range of multiplex applications screening for cancer biomarkers, electrolyte imbalance, and proteins, etc. This review focuses on electrochemical detection methods as they have progressed more than optical techniques for wearable applications. The approach taken is to review the three main flexible substrate categories: (i) paper/paper hybrids, (ii) synthetic polymers, and (iii) fabrics as reported in the literature and to highlight multiplex biomarker combinations demonstrated with each substrate type.

\section{Paper/hybrid devices}

Cellulose is an abundant, natural, low-cost polymer (trees, plants, bacteria, algae) extensively used in bioassays. ${ }^{\text {[33] }}$ Paper assays generate significant research interest, they are

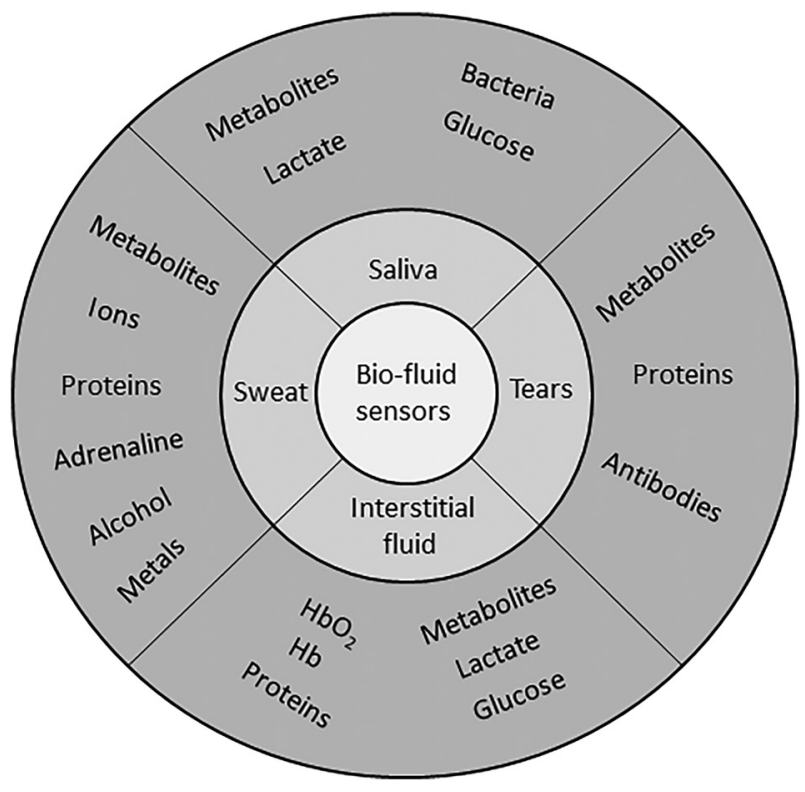

Figure 1. Overview of target biomarkers within saliva, tear, sweat, and interstitial fluid (ISF), to be monitored at the eye, skin, and mouth locations with potential for multiplex combinations. inexpensive, easy to use, flexible, consume low reagent volume, and deliver rapid results. ${ }^{[34-36]}$ In this section, we highlight paper and hybrid/paper devices applied to multiplex biomarker monitoring, in applications including cancer, metabolites, and pathogen detection. Microfluidic paper-based analytical devices (mPADs) is a term used for paper and hybrid test devices. ${ }^{[37-41]}$ Capillary action makes paper an ideal material for wearable/point-of-care applications, avoiding the need for pumps, as local surface modification [e.g., wax, ${ }^{[42]}$ polydimethylsiloxane (PDMS) $\left.{ }^{[43]}\right]$ manipulates sample flow to reaction sites. Tests are primarily based on enzyme-linked immuno-assays (ELISA), where reagents can be directly adsorbed onto porous paper at reaction sites. Electrochemical detection is extensively used in low-cost printing techniques (inkjet) that dispense materials forming electrode patterns. Electrochemical detection is extensively used in portable systems due to low-power consumption and simple instrumentation, a significant advantage over optical systems.

\section{Glucose, lactose, uric acid}

Assays incorporating catalytic reactions and identifying multiple analytes have been demonstrated on paper substrates. A multiplex electrochemical device was demonstrated for glucose, lactose, and uric acid detection in human serum samples. ${ }^{[4]}$ Electrodes were screen printed from carbon ink containing Prussian blue (PB) for the working electrode (WE) and the counter electrode (CE); the reference electrode (RE) was $\mathrm{Ag} / \mathrm{AgCl} . \mathrm{PB}$ is extensively incorporated as a mediator into electrochemical assays, facilitating electropotential shift to mitigate against competing biomolecules. Test areas were prepared by spotting $0.3 \mu \mathrm{L}$ of GOx, lactate oxidase (LOx), and uricase solutions onto WE areas. Chrono-amperometric detection was used to monitor enzyme reactions within each target zone, at a sampling rate of 10 $\mathrm{Hz}$. PB, as an electrode mediator, reduced catalytic reaction potentials over the range -0.2 to $0.2 \mathrm{mV}(\mathrm{Ag} / \mathrm{AgCl})$ minimizing interference from uric and ascorbic acid. Detection was based on the reduction of $\mathrm{H}_{2} \mathrm{O}_{2}$ at $0 \mathrm{~V}$. The limits of detection (LODs) were: glucose $0.21 \mathrm{mM}$ (range $0-100 \mathrm{mM}$ ), lactate $0.36 \mathrm{mM}$ (range $0-50 \mathrm{mM}$ ), and uric acid $1.38 \mathrm{mM}$ (range 0 $35 \mathrm{mM}$ ) in human serum. Direct enzyme immobilization on WEs is a popular approach for electrochemical sensor implementation. However, incorporating three-dimensional (3D) structures can improve the performance and enhance specificity. A hydrogel-paper hybrid assay demonstrated the glucose and protein detection in urine. ${ }^{[35,45]}$ Microfluidic channels were defined by patterning hydrophilic paper with hydrophobic polymer for controlled liquid flow, delivering a low-cost approach for multiplex biomarker detection. The paper substrate was soaked in SU8 polymer solution; following UV curing, non-cross-linked polymer was removed in a propylene glycol monomethyl ether acetate solution. Three-dimensional hydrogels enhance reagent immobilization and assay sensitivity, through increased target capture and optimizing enzyme activity. ${ }^{[46]}$ A novel screen-printed mPAD, with all-carbon 
Table I. Examples of common target biomolecules, sample fluid, and clinical concentration ranges used as applications for wearable health-monitoring systems.

\begin{tabular}{|c|c|c|c|c|}
\hline Biomarker & Sample type & Health condition & Range & Ref. \\
\hline Glucose & Saliva, sweat & Diabetes & $0.5-1.6 \mathrm{mM}$ & [24] \\
\hline Glucose & Tear & Diabetes & $0.025-1.475 \mathrm{mmol} / \mathrm{L}$ & [25] \\
\hline Proteins & Sweat and saliva & Disease screening & $\mathrm{ng} / \mathrm{mL}-\mathrm{pg} / \mathrm{mL}$ & [1] \\
\hline Electrolytes & Sweat & Dehydration & $0-110 \mathrm{Mm}$ & [26] \\
\hline Interleukin 6 & Sweat & Inflammation & $0.02-20 \mathrm{pg} / \mathrm{mL}$ & [27] \\
\hline Lactate, salts & Saliva & Dehydration & $0-110 \mathrm{mM}$ & [5] \\
\hline $\mathrm{Zn}, \mathrm{Cd}, \mathrm{Pb}, \mathrm{Cu}, \mathrm{Hg}$ & Sweat & Heavy metal poisoning & $100-300 \mu \mathrm{g} / \mathrm{L}$ & [28] \\
\hline Potassium & Sweat and saliva & Hypo and hyper kalemia & $3.6-5.2 \mathrm{mmol} / \mathrm{L}$ & [29] \\
\hline Alcohol/ethanol & Sweat & Intoxication & $0-36 \mathrm{Mm}$ & [30] \\
\hline Cortisol & Saliva and sweat & Hypertension & $7-28 \mu \mathrm{g} / \mathrm{dL}$ & [31] \\
\hline Pathogen cells & Sweat/urine & Infectious disease & $10-100$ cells $/ \mathrm{mL}$ & [32] \\
\hline
\end{tabular}

electrode-enabled electrochemical assay (SP-ACE-EC- $\mu$ PAD), simultaneously detected glucose and uric acid in urine. ${ }^{[4]}$ Carbon ink electrodes were deposited on the substrate using low-cost screen printing. GOx and uricase were deposited in reservoir locations by spotting $2 \mu \mathrm{L}$ of enzyme solution, followed by air drying $(20 \mathrm{~min})$.Glucose and uric acid were detected in urine using chrono-amperometry, providing fast and accurate results. Spiked glucose $(0.25,0.5,0.75 \mathrm{Mm})$ and uric acid $(0.1,0.2,0.3 \mathrm{mM})$ samples $(20 \mu \mathrm{L})$ were evaluated on the device, delivering results within $3 \mathrm{~min}$. This simple detection approach applied $300 \mathrm{mV}$ step potential with current monitored over time and was highly suitable for wearable applications.

\section{Proteins}

Color change detection is an instrument-free approach, extensively used with lateral flow assays. Such tests usually identify a single target. In a novel approach of multiplex diagnostics, hydrogel was formed on a paper substrate using an aptamer cross-linker, trapping glucoamylase (GA). ${ }^{[4]}$ Glucose detection, based on enzymatic oxidation of iodide to iodine, altered test spot color from clear to brown. For protein detection, spots changed from yellow to blue following tetrabromophenol blue ionization. The device used a target-responsive aptamer crosslinked hydrogel, for selective target recognition. With the target present, the hydrogel collapsed releasing GA into the solution and amylose hydrolyzed by GA generated glucose as the liquid progressed along the paper. A catalytic GOx reaction along the channel converted glucose to gluconic acid and $\mathrm{H}_{2} \mathrm{O}_{2}$, resulting in a brown color change as horse radish peroxidase (HRP) catalyzed poly(DAB) from colorless 3,3'-diaminobenzidine (DAB). The resulting color change length along the test strip correlated with target concentrations. The flexibility of the hydrogel-aptamer structure facilitated multiple target detection in urine, i.e., glucose $(0.7-10.5 \mathrm{mM})$, cocaine $(0-100 \mu \mathrm{M})$, and adenosine detection ( 0 to $800 \mu \mathrm{M})$. Adenosine is a cancer biomarker used to monitor disease progression. ${ }^{[49]}$ While colorbased detection is fast, results are subjective and may suffer from reduced sensitivity. The authors highlight how distancebased color detection is less subjective compared with spot color change, and performance was comparable to commercially available dipstick tests.

\section{Cell targets}

Electrochemical luminescence (ECL) combines electrochemical activity with optical detection by incorporating a chemiluminescent molecule into the ELISA. This approach is useful for quantitative detection and gives enhanced sensitivity over purely color-based assays. Conventionally, ECL assays are implemented in microwells or on microfluidic devices; however, paper-based ECL has been demonstrated. A hybrid paper-PDMS device was manufactured for rapid multiplex pathogen detection. ${ }^{[32]}$ The hybrid approach offered a simple, biocompatible, 3D material for reagent storage and immobilization, without complex surface chemistry, while PDMS microfluidics defined reaction zones. Fluorescent aptamers functionalized with graphene oxide (GO) were deposited by pipette on chromatography paper. The aptamer-GO solution was adsorbed onto paper defining a probe microarray, screening for target pathogens. GO in close proximity to a fluorescence probe caused quenching, thus switching CY3-labeled aptamers to an "off" state. The aptamer became rigid with specific target binding, increasing GO-Cy3 separation, switching "on" fluorescence signal by reducing quenching. The simple test required sample loading followed by incubation (10 min), without washing prior to fluorescence detection. This approach 
offered direct pathogen-specific detection without nucleic acid screening techniques (e.g., PCR). Lactobacillus acidophilus detection was demonstrated over the range $0-300 \mathrm{cfu} / \mathrm{mL}$ with an estimated LOD of $11 \mathrm{cfu} / \mathrm{mL}$. Multiplex pathogen detection was demonstrated for Staphylococcus aureus and Salmonella enterica, achieving good specificity (Fig. 2). The detection range for $S$. enterica was $42.2-675.0 \mathrm{cfu}$ and for $S$. aureus $104-106 \mathrm{cfu} / \mathrm{mL}$, with LOD of $61.0 \mathrm{cfu} / \mathrm{mL}$ (S. enter$i c a)$ and $800.0 \mathrm{cfu} / \mathrm{mL}$ (S. aureus). Performance was comparable to cell culture and molecular diagnostic approaches.

A novel laboratory-on-paper-based chemiluminescence assay was demonstrated for cancer biomarkers. ${ }^{[50]}$ Reaction chambers were formed by screen printing hydrophobic wax layers into porous paper, with covalent immobilization of capture antibodies using a glutaraldehyde linker molecule. The assay consisted of: (i) immobilizing sample-specific capture antigens, (ii) addition of HRP-labeled signal antibodies to detection zones, and (iii) injection of luminol-p-iodophenol$\mathrm{H}_{2} \mathrm{O}_{2}$, triggering chemiluminescence. Incubation time was optimized at $210 \mathrm{~s}$. Increasing concentrations of three tumor markers were evaluated in phosphate-buffered saline buffer, delivering linear ECL responses for each target range: (i) $\alpha$-fetoprotein (AFP) $0.1-35.0 \mathrm{ng} / \mathrm{ml}$, (ii) carcinoma antigen 125 (CA125) 0.5-80.0 U/mL, and (iii) carcinoembryonic antigen (CEA) $0.1-70.0 \mathrm{ng} / \mathrm{mL}$. The LODs of the three targets were within clinically acceptable limits when evaluated with human serum, in agreement with a commercial ECL cancer test. A paper-based ECL assay was also implemented screening for four cancer biomarkers: AFP, CA125, carcinoma antigen 199, and CEA. ${ }^{[51]}$ ECL detection was demonstrated in human serum using TPA [tris-(bipyridine)-ruthenium(II) $[\mathrm{Ru}(\mathrm{bpy})$ 32p]-tri-n-propylamine]. Eight reaction chambers with feeder channels were defined by wax printing. Eight WEs incorporated into the device stimulated ECL during voltage sweeps

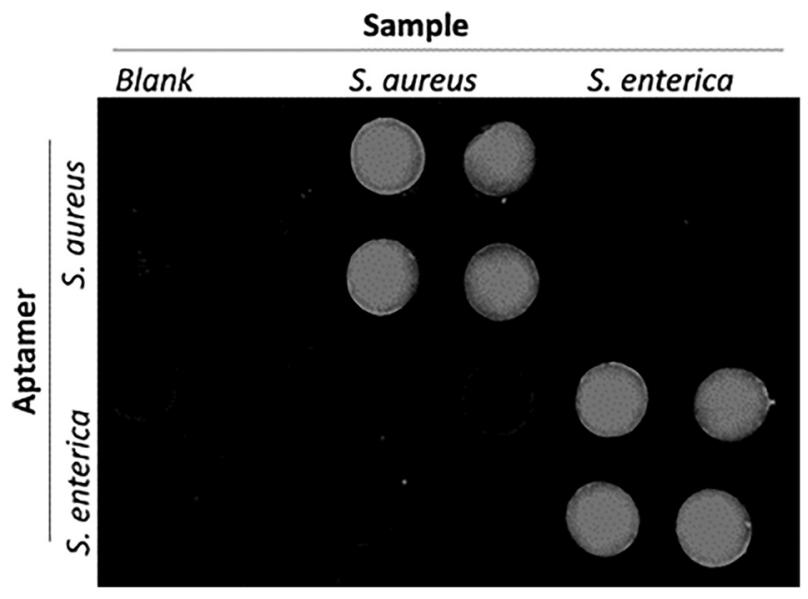

Figure 2. A cross-reaction assay incorporating Staphylococcus aureus $\left(10^{6}\right.$ $\mathrm{cfu} / \mathrm{mL}$ ) and Salmonella enterica (1375 cfu/mL) demonstrates the selectivity of the sensor to multiple pathogen targets for each immobilized aptamer (reproduced from Ref. 32 with permission from Royal Society of Chemistry).
(0.5-1.1 V). For manufacture, fluidics were first fabricated followed by electrode screen printing (Carbon WE, $\mathrm{Ag} / \mathrm{AgCl}$ reference). Capture antibodies $(2 \mu \mathrm{L}, 20 \mathrm{mg} / \mathrm{mL})$ were immobilized on each WE using chitosan coating and GA cross-linking. For target capture, sample was added to each electrode and incubated for $30 \mathrm{~min}$. ECL detection was realized by adding TPA $(0.01 \mathrm{mM})$ and monitored during voltage sweeps. The test was evaluated with blood serum from high-risk cancer patients; measured biomarker concentrations agreed with commercial cancer test results. An origami-like paper device was developed to simultaneously screen for four cancer cells (MCF-7, HL-60, K562, CCRF-CEM). ${ }^{[52]}$ Target-specific aptamers were immobilized on gold electrodes, and porous AuPd nanoparticles labeled with concanavalin-A acted as probes. The nanoparticles selectively bound to mannose on the captured cell surface. Carbon WEs were screen printed in each capture reservoir with a single $\mathrm{Ag} / \mathrm{AgCl} \mathrm{RE}$. Au nanoparticles were grown on WE surfaces before aptamer modification, forming an $\mathrm{Au}$-thiol monolayer. Wax channel and chambers were defined for liquid handling. For detection, test solutions $(10 \mu \mathrm{L})$ were delivered to each chamber and incubated for $15 \mathrm{~min}$. After cell capture, the bio-conjugate solution (AuPd@Con-A) was added labeling captured cells. ECL measurements were performed by sweeping voltage $(-0.3$ to $-1.8 \mathrm{~V}$, scan rate $100 \mathrm{mV} / \mathrm{s}$ ) on each electrode while the monitoring optical signal. A log relationship existed between ECL signals and cell concentration (working range 450-10 $0^{5}$ cells/ $\mathrm{mL}$ ) demonstrating potential for low-cost, rapid cancer cell screening. The test exhibited good specificity, with a slight signal increase, to non-targeted cancer cells. Test variation was $<4 \%$ [coefficient of variation $(\mathrm{CV})$ ] and devices were viable for up to 5 weeks. Similar assays were also developed for specific cancerous cell screening. ${ }^{[53,54]}$ Paper stacking was demonstrated as a novel approach for assay implementation. ${ }^{[55]}$ Paper sheets, pre-incubated with biologic reagents, were skived into multiple test sheets facilitating mass device manufacture for multiplex applications. The width of a single paper sheet formed each reaction site, with multiple sheets assembled to implement multiplex barcode assays. Test readout was performed using a commercial barcode scanner and simultaneously distinguished positive results for HBV, HCV, HIV, TP over negative samples. Barcode assays were fabricated by gluing white paper, red paper, and paper immobilized with capture probes together in a defined manner. Lateral flow delivered target to immobilized capture probes, while AuNPs labels changed paper color (white to red) indicating a positive test. Devices evaluated with human samples demonstrated reproducible and specific virus detection at clinically relevant levels. An emerging area is paper-based molecular diagnostics using amperometric $^{[56]}$ and impedance ${ }^{[57]}$ detection; however, multiplex assays using these detection methods on flexible substrates have yet to be demonstrated. A challenge for paper assays is valved fluidic control; a novel paper device (Fig. 3) incorporated active electromagnetic valving and timed incubation. Active on paper valving was implemented as a sample wicked 

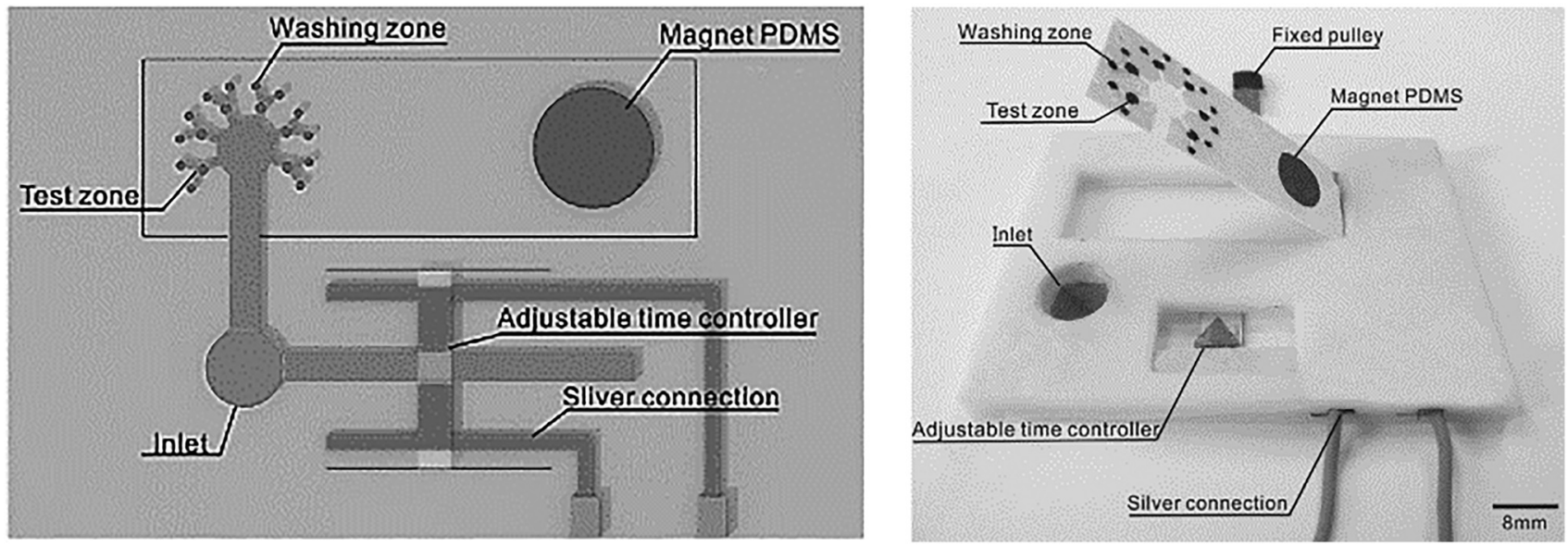

Figure 3. Programmed incubation time and active electromagnetic valving were implemented on a paper-based ECL assay, screening for protein cancer biomarkers $\alpha$-fetoprotein (AFP) and carcinoma antigen 125 (CA-125) in human serum sample (reprinted from Ref. 58 Copyright Sensors \& Actuators B).

between two electrodes, completing a circuit to activate electromagnetic valves. The device screened for multiple cancer biomarkers and programmed incubation times facilitated specific protein detection. Significant progress has also been made with mPAD multiplex devices, screening for specific biomarker combinations. Catalytic detection is the most popular technique adapted with mPADs assays, and aptamers are easily incorporated to enhance selectivity and sensitivity. ECL has enhanced signal benefits over visual color change assays, and electrochemical-based techniques offer potential for portable point-of-care and wearable biomarker detection. Challenges for paper-based assays include long-term biomolecule activity with refrigeration required to maintain viable assays. Paper assay manufacture is low cost and easy to implement in developing countries; however, humidity and temperature variation can impact reproducibility. Capillary flowrates can be modified by change in sample viscosity due to medication; thus, internal controls are required for test verification. Wearable paper devices have primarily focused on monitoring interstitial fluid and sweat constituents (e.g., electrolytes, glucose, lactate) using simple assays, while point-of-care paper applications extend to urine, saliva, and blood biomarkers. A future step will be to incorporate complete sample preparation and detection into a single test.

\section{Polymer substrates}

A diverse range of flexible synthetic polymer materials are available for sensor applications including polyimide (PI), polyethersulfone, polyetheretherketone, poly(ethylene napthalate), polycarbonate, and polyethylene terephthalate (PETE). Such materials are compatible with mass manufacture methods for thin flexible substrates (e.g., spin coating, extrusion, injection moulding). They are compatible with processing steps to deposit electroactive layers (organic or inorganic) for device fabrication [electrode, field effect transistor (FET), nanowire] and biochemistry protocols immobilizing capture biomolecules (antibodies, DNA) or enzymes, for target capture/recognition. Electroactive materials incorporated into flexible sensors include conductive polymers (polypyrrole, PANI, polythiophene), semiconductor materials $\left(\mathrm{ZnO}, \mathrm{In}_{2} \mathrm{O}_{3}\right.$, graphene), and nanowire/nanoparticle materials (carbon nanotubes). Microelectronic deposition techniques (chemical vapor deposition, evaporation) are extensively used to realize pure films defining devices and electrical contacts. Approaches where prepolymer liquids incorporate nanowire/nanoparticle materials have been implemented by electrospinning, micro-contact printing, spin-coating, etc. Such approaches produce robust devices amenable to substrate bending, twisting, and stretching.

\section{Glucose, electrolytes, lactate}

A flexible substrate for multi-target (glucose, lactate, $\mathrm{Na}^{+}, \mathrm{K}^{+}$) sweat screening was demonstrated for exercise monitoring. ${ }^{[59]}$ Sensors were fabricated on flexible polyethylene terephthalate (PET) substrates conforming to skin contour. Gold electrodes were deposited on the substrates using a lift-off process. Enzymes (GOx, LOx) immobilized on the substrate using a polysaccharide chitosan membrane facilitated amperometric measurement. Ion-selective electrodes (ISEs) incorporating PEDOT:PSS as an ion-to-electrode transducer facilitated selective $\mathrm{Na}^{+}$and $\mathrm{K}^{+}$detection. The $\mathrm{RE}(\mathrm{Ag} / \mathrm{AgCl})$ was coated with a polyvinylbutyral (PVB) membrane containing carbon nanotubes enhancing electrical properties. Such coatings facilitate long-term measurements and minimize signal drift. Incorporating $\mathrm{PB}$ dye shifted reduction potentials to $0 \mathrm{~V}$ removing the need for additional sensor power, an important consideration for wearable devices. The ion sensors demonstrated detection over physiologically relevant ranges $\left(\mathrm{K}^{+}\right.$ $10-160 \mathrm{mM}$ and $\mathrm{Na}^{+} 1-32 \mathrm{mM}$ ). Temperature variation was found to have a significant impact on enzyme activity, and thermal compensation addressed overestimation in glucose and 
lactate measurements. The system was evaluated during moderate activity on an exercise bike. After perspiration onset, decrease in measured glucose and lactate levels was observed; however, lactate levels stabilized as expected with continuous steady exertion. $\mathrm{Na}^{+}$increased and $\mathrm{K}^{+}$decreased as perspiration commenced and stabilized as exercise continued. When applied to different body locations (forehead, wrist), the system recorded different trends due to varied sweat volumes and skin characteristics. During high-intensity exercise, measured trends varied across four volunteers for $\mathrm{Na}^{+}$and $\mathrm{K}^{+}$. During intense exercise, observed glucose trends were well correlated across subjects, however lactate levels varied between subjects. Highlighting how large scale population testing is required to establish biomarker relevance. A fully integrated autonomous platform (Fig. 4) ${ }^{[60]}$ was developed to quantify $\mathrm{Na}^{+}, \mathrm{Cl}^{-}$, and glucose in sweat samples for cystic fibrosis (CF) and diabetes monitoring. Iontophoresis electrodes coated with pilocarpineloaded hydrogel were incorporated to locally stimulate sweat glands in sedentary patients. The measurement consisted of two phases: (i) application of a local current to generate sample and (ii) parameter measurement using ISEs.

Ag electrodes were deposited on a PET substrate facilitating mechanical flexibility for direct skin contact. An Ag/AgCl electrode for $\mathrm{Cl}^{-}$detection was deposited by modifying an $\mathrm{Ag}$ electrode with a $\mathrm{FeCl}_{3}$ solution. The $\mathrm{Na}^{+}$detection electrode was realized by depositing a $\mathrm{Na}^{+}$-selective layer upon an $\mathrm{Ag}$ electrode. PVB coatings saturated with chlorine ions were applied to formulate REs. For CF diagnostics, the system was evaluated by measuring sweat chloride concentration. Levels $>60 \mathrm{mM}$ indicated increased probability of disease. Measurements were made on three CF patients and six healthy volunteers; the average $\mathrm{Na}^{+}$and $\mathrm{Cl}^{-}$for $\mathrm{CF}$ sufferers were 82.3 and $95.7 \mathrm{mM}$, respectively, while average healthy readings were 26.7 and $21.2 \mathrm{mM}$. For glucose measurements, a correlation between blood and sweat levels was demonstrated on a group of fasting and post fasting volunteers. A skin-mounted microanalytical flow system was demonstrated ${ }^{[61]}$ to monitor sweat lactate and glucose levels during light indoor cycling sessions. An optimized microfluidic sweat collection device was designed to deliver sweat sample collected from the skin to measure electrodes. This was the first time a microfluidic interface was optimized for sample collection on a skin wearable sensor. The device structure consisted of two PDMS layers, one incorporating the electrode substrate and the second defining fluidic channels. Gold electrodes (WE, CE) were modified with $\mathrm{PB}, \mathrm{GOx}$, and LOx for detection. The device also incorporated an $\mathrm{Ag} / \mathrm{AgCl} \mathrm{RE}$. When evaluated with spiked artificial sweat sample, flowing at $200 \mathrm{~mL} / \mathrm{min}$, standard deviations of 1.2 and $1.6 \%$ were recorded for lactate $(12 \mathrm{mM})$ and glucose $(10 \mathrm{mM})$. The linear operating ranges for lactate was 4-20 $\mathrm{mM}$ and for glucose $2-10 \mathrm{mM}$. Detection sensitivity was $29.6 \mu \mathrm{M} / \mu \mathrm{A}$. On body tests were undertaken with two healthy subjects over $20 \mathrm{~min}$ of moderate indoor cycling. Different signals were obtained compared with in vivo tests due to temperature, $\mathrm{pH}$, and flowrate variation. Similar measured metabolite trends were previously reported. ${ }^{[59,62,63]}$ Flexible conformal biosensor arrays were manufactured using printed ultrathin metal oxide semiconductor technology for $\mathrm{pH}$ and glucose detection. ${ }^{[64]}$ Arrays were manufactured from indium oxide films $(3.5 \mathrm{~nm})$ with low impurity concentration. The metal oxide films were robust to stress/strain caused by substrate bending, twisting, and stretching. The surface was easily
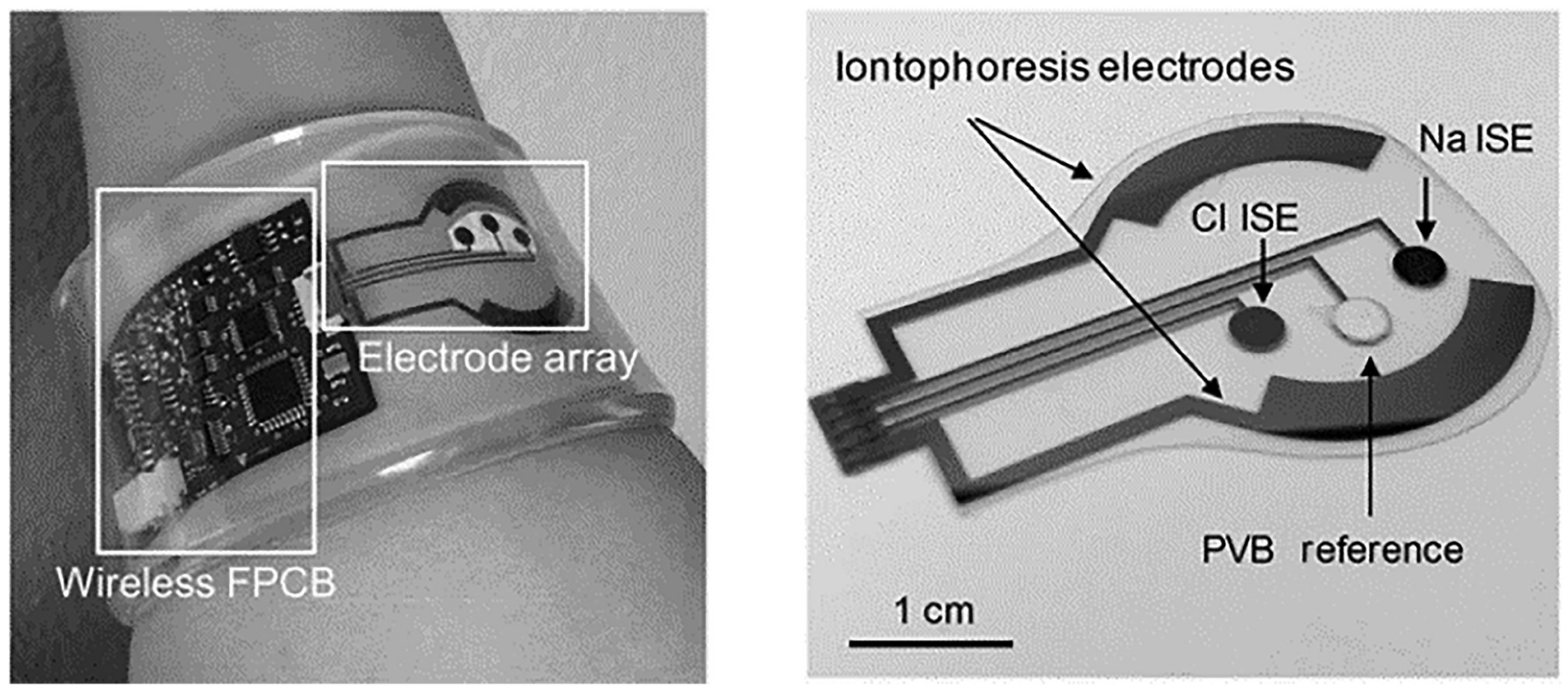

Figure 4. The flexible patch-type system incorporating electrodes and electronics for wireless $\mathrm{Na}^{+}, \mathrm{Cl}^{-}$, and glucose monitoring in sweat sample (reprinted from Ref. 60 Copyright PNAS 2017). 
modified for biomolecule immobilization, and the sensor demonstrated both $\mathrm{pH}$ and glucose detection for diabetes and wound monitoring. Flexible FET devices were formed on ultrathin PI films $(2 \mu \mathrm{m})$; the active layer $\left(\operatorname{In}_{2} \mathrm{O}_{3}\right)$ was spin coated and then annealed at $250{ }^{\circ} \mathrm{C}$. Interdigitated electrode arrays were patterned on the metal oxide film defining metal contacts. Sensor thin films were released from support substrates by delamination in water, facilitating stretching and unfolding. Protonation of surface hydroxyl groups and primary amines of aminopropyltriethoxysilane occurred on the metal oxide surface, as $\mathrm{pH}$ decreased from $\mathrm{pH} 5.5$ to $\mathrm{pH}$. For glucose detection, GOx immobilized on FET surfaces produced hydrogen peroxide from glucose oxidation. Measured currents were recorded over a clinically relevant range $(100-400 \mu \mathrm{M})$, and devices were suitable for simultaneous on-skin glucose and $\mathrm{pH}$ measurements. Flexible organic electrochemical transistors (OECTs) formed on a flexible substrate demonstrated simultaneous detection of uric acid and glucose. ${ }^{[65]}$ The device was formed by the deposition of electroactive PEDOT:PSS layers on a flexible PET substrate $(50 \mu \mathrm{m})$ with platinum contact electrodes. Flexible devices could be attached to conformal surfaces and were robust to repeat bending cycles (1000 times). The device was sensitive to $\mathrm{H}_{2} \mathrm{O}_{2}$ formed during enzymatic reactions. To overcome the sensitivity of the platinum gate electrodes to interfering molecules [e.g., dopamine, glucose, uric acid (UA), and ascorbic acid], electrodes were modified with a bilayer consisting of graphite flakes and Nafion, then encapsulated with a conductive polymer (PANI). After surface modification, positively charged molecules (e.g., dopamine) were repelled from the surface (Nafion repelled UA and ascorbic acid), while larger molecules (e.g., glucose) could not penetrate the PANI nanometer-sized pores. Gate modification had minimal impact, as $\mathrm{H}_{2} \mathrm{O}_{2}$ penetrated the bilayer delivering good detection (LOD $10^{-9}$ ). Uricase (UOx) was immobilized on PANI using GO and oxidation of uric acid also generated $\mathrm{H}_{2} \mathrm{O}_{2}$. A physiologically relevant response range was recorded $\left(100 \times 10^{-9}-500 \times 10^{-6} \mathrm{M}\right)$ for uric acid. The device detected UA in saliva at $173 \pm 20 \times 10^{-6} \mathrm{M}$ and saliva glucose in a healthy individual $\left(103 \pm 10 \times 10^{-6} \mathrm{M}\right)$. The bilayer blocking effect was relevant only to potentiometric sensors as fields associated with amperometric detection negated bilayer charge blocking at the device surface. Blocking layer efficiency is an important parameter along with electrode poisoning, requiring consideration in catalytic sensor methods. A flexible multisensor patch was fabricated to simultaneously monitor sodium, $\mathrm{pH}$, and lactate sweat during exercise sessions. ${ }^{[66]}$ The patch adhered to skin on the lower back where a flexible microneedle array and microfluidic channel collected sweat sample for electrochemical measurement. The innovative sampling mechanism continuously drew sample across the sensors for real-time detection. The overall patch thickness was $180 \mu \mathrm{m}$ facilitating comfortable long-term application. For $\mathrm{Na}^{+}$detection, a poly(vinylchloride) ion-selective membrane was drop cast onto Pt/PEDOT electrode gasket, the RE was prepared by doping the polymeric membrane with a lipophilic salt. For lactate detection, the RE was treated with potassium dichromate. For $\mathrm{pH}$ detection, iridium oxide was electrochemically deposited on electrodes by chemical oxidation. LOx drop cast onto electrodes coated with sulphonated polyether-ether sulphone:polyethersulphone for selective lactate detection. Sensor signals were wirelessly transmitted to a mobile phone for data analysis. The system was evaluated on six male volunteers during exercise sessions. Temperature recalibration was applied to the sensors compensating for modified enzyme activity. Saliva samples were taken during exercise to determine cortisol concentration using a commercial immunoassay. Measured sodium, lactate, and $\mathrm{pH}$ levels were in agreement with the previous reported studies and in line with clinical levels for exercise sessions and were repeatable with CVs of $6 \%$ (Na), $7 \%(\mathrm{pH})$, and $9 \%$ (lactate). The sensors were robust to interfering sweat constituents, e.g., uric acid, ascorbic acid, and glucose. A disposable patch incorporating microfluidics and assay reagent implemented a passive test screening for $\mathrm{Cl}, \mathrm{Zn}$, and Na. ${ }^{[67]}$ The device was composed of three layers: (i) adhesive, (ii) microfluidic, and (iii) light shield. Microfluidic channels, valves, and chambers realized a passive pump mechanism delivering sweat sample from skin to assay reservoirs. Fluorescent probes reported target concentrations, with signals read using a smartphone via miniaturized optical fluorescence system. The fluidic system (Fig. 5) contained check valves to facilitate timed delivery of sweat sample to each reaction chamber once a threshold liquid pressure was achieved. The light shield layer protected the probes during skin application for sweat sampling and assay reaction. For $\mathrm{Na}$ and $\mathrm{Zn}$ detection, there was a direct relationship between intensity and target concentration correlating with physiologically relevant ranges $(\mathrm{Na} 20-60 \mathrm{mM}, \mathrm{Zn} 1.4-27 \mu \mathrm{M})$. An inverse fluorescent signal was correlated with increasing $\mathrm{Cl}$ sweat content over the physiologic range 20-60 mM.

\section{Alcohol, glucose}

A flexible sensor was developed to simultaneously monitor alcohol and glucose with low sample volume $(1-3 \mu \mathrm{L})$; this combination facilitated a wearable system to monitor correlation between alcohol consumption and diabetes. ${ }^{[68]}$ Flexible porous PI membranes were used as a substrate to fabricate zinc oxide electrodes. The porous polymer membrane wicked sweat away from the skin to the sensor surface. Selective biomolecule transport through the porous membrane enhanced the signal by minimizing interference from ions and lipids. The sensor was evaluated on synthetic sweat spiked with glucose and ethanol over clinically relevant ranges, with impedance measured over the frequency range $50-500 \mathrm{~Hz}$. Variation in impedance due to electron charge transfer and electrical double-layer modulation modified electrode capacitance as target biomolecule concentration varied. Changes in imaginary impedance correlated with ethanol concentration over the range $0.01-200 \mathrm{mg} / \mathrm{dL}$. Sweat $\mathrm{pH}$ variation $(\mathrm{pH} 4-8)$ had an impact on ethanol estimates. The sensor also demonstrated selectivity against interfering biomolecules (e.g., uric acid, 


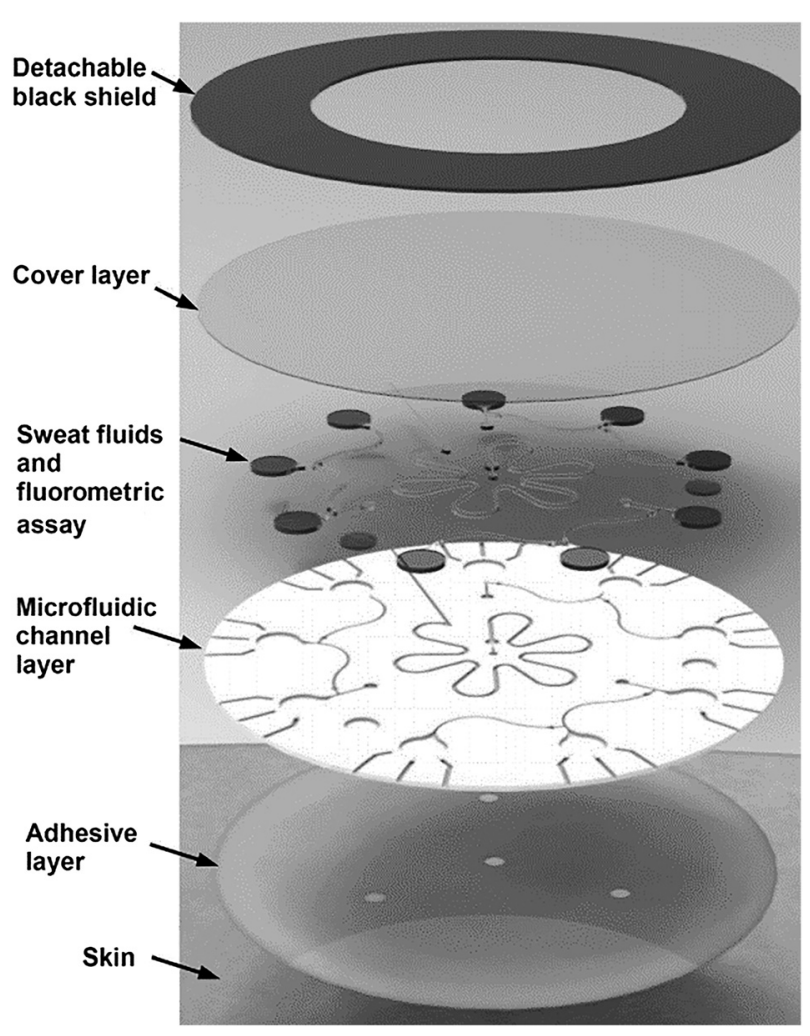

Figure 5. A multilayer patch incorporating adhesive, microfluidics, and assay reagents was demonstrated for the detection of $\mathrm{Cl}$, Na, and $\mathrm{Zn}$ over clinically relevant ranges (reproduced from Ref. 67 with permission from Royal Society of Chemistry).

glucose, lactate, creatine, etc.). For combinatorial experiments, glucose measurements were made at $100 \mathrm{~Hz}$, and the performance of glucose and alcohol measurements were comparable to commercial devices for glucose (Accu-Chek ${ }^{\circledR}$ ) and alcohol (BACtrac $\left.{ }^{\circledR}\right)$ and within acceptable limits of error.

\section{Heartrate, lactate}

Combining electrophysiologic and biochemical measurements on a single patch ${ }^{[69]}$ augmented heartrate monitoring with lactate measurements, offering a more comprehensive fitness monitor compared with electrophysiologic measurements alone. The system was composed of a three electrode lactate sensors and a bipolar electrocardiogram sensor fabricated on a flexible substrate for skin adhesion. The device was fabricated by screen printing electrodes on a flexible polyester sheet. Portable instrumentation incorporated a potentiostat and an electrocardiogram with Bluetooth telemetry. The hybrid device was tested on three subjects during cycling exercise sessions. The patch size $(7 \mathrm{~cm} \times 2 \mathrm{~cm})$ was set by electrocardiogram electrode separation. Placing the patch on the chest region was optimal for heartrate monitoring and also generated sufficient sweat sample for lactate measurement. The lactate electrodes were fabricated between the heartrate electrodes. PB ink was used to print the WEs, which were highly sensitive to hydrogen peroxide produced by enzymatic lactate oxidation. The $\mathrm{Ag} / \mathrm{AgCl} \mathrm{RE}$ was also screen printed. The sensors were separated by an ecoflex hydrophobic layer preventing signal distortion through multiple electrical pathways thus minimizing cross-talk between sensors. Results from continuous cycling highlighted heartrate measurements between $60 \mathrm{bpm}$ (resting) and $120 \mathrm{bpm}$ (full exertion); at exercise commencement, no variation in lactate was measured on the lactate sensor due to lack of perspiration; however, with sweat onset, the LOx sensor shows signal increase correlating with heartrate and exertion. Dilution of sweat lactate content after a period of time due to profuse sweating was also detected by the sensor. During exercise cooldown, the heartrate returned to normal and the lactate readings reduced as expected under normal clinical conditions.

\section{Heavy metals}

Metal detection is well established in electrochemistry ${ }^{[70]}$ and has been applied to environmental, ${ }^{[71]}$ agriculture, ${ }^{[72]}$ and health $^{[73]}$ applications. Portable amperometric technology is suitable for system miniaturization. ${ }^{[74]}$ Metal screening in wearable health devices has emerged as an area of interest as metal deficiency (e.g., $\mathrm{Cu}, \mathrm{Zn}$ ) causes disease, ${ }^{[28,75]}$ while metal build-up has negative health implications. Screening sweat for heavy metals using electrochemical techniques ${ }^{[76,77]}$ has been adapted by wearable technologies. ${ }^{[78]}$ Heavy metal detection ( $\mathrm{Zn}, \mathrm{Cd}, \mathrm{Pb}, \mathrm{Cu}, \mathrm{Hg}$ ) in body fluids was demonstrated using square wave anodic stripping voltammetry on $\mathrm{Au}$ and $\mathrm{Bi}$ electrodes. ${ }^{[79]}$ A five-electrode microarray was evaporated and patterned (lift-off) on a PET substrate, then coated with a Nafion protective layer. ${ }^{[80]}$ A PDMS sample reservoir (20$30 \mu \mathrm{L}$ ) was sealed on the sensor substrate for sweat sample retention, providing a stable sensor liquid measurement interface and highlighting how microfluidic designs can be incorporated with flexible sensor substrates to enhance biomarker detection.

\section{Proteins and DNA}

Single-target immunoassays have limited diagnostic value as many biomarkers (e.g., proteins) are associated with a number of conditions (e.g., cancers); thus, there is a significant interest in implementing multiplex tests to improve diagnosis accuracy. Gold WE microarrays were deposited on flexible PI substrates for cytokine detection using impedance measurements. ${ }^{[81]}$ The same group fabricated poly(pyrrole) (PPy) microwires (PPY $\mu \mathrm{Ws}$ ) on flexible substrates including PETE, cyclic olefin copolymer, polyethylene naphthalate, and PI using microcontact printing $(\mu \mathrm{CP}) .{ }^{[82]}$ The flexible sensors demonstrated multiplex cytokine detection by impedance spectroscopy. PPy is a good conductive polymer for electrical biosensors due to its electrical conductivity, environmental stability, biocompatibility, and easy synthesis. ${ }^{[83]}$ Human interleukin-10 antibodies were chemically immobilized on PРy $\mu$ Ws using gluteraldehyde cross-linker. Impedance spectroscopy was used to detect rhIL-10 biomarkers over the range $1-50 \mathrm{pg} / \mathrm{mL}$, with 
sensitivity $0.026(\mathrm{pg} / \mathrm{mL})$. This group also devised a PDMS $\mu \mathrm{CP}$ process defining PPy nanowires on flexible thermoplastic surfaces (PETE, polyether ether ketone), by covalent bonding. Carbodiimide cross-linker chemistry attached specific monoclonal antibodies (anti-human IL-6) to diazonium functionalized nanowires, defining impedance sensors for IL-6 detection. The LOD was $0.013 \mathrm{pg} / \mathrm{mL}$ with a linear operating range $1-50 \mathrm{pg} / \mathrm{mL}$ and demonstrated potential for multiplex cancer screening using IL-6 antibody biomarkers. Target-specific aptamers were combined with nanographene oxide (NGO) sensor arrays to selectively screen for nine cancer-related proteins using fluorescence detection ${ }^{[84]}$ at nanomolar target concentrations. Multiple protein targets were also detected using aptamer/NGO substrates with NGO-modifying polymer substrate elasticity. ${ }^{[85]} \mathrm{NGO}$ also demonstrated superior limits of detection over graphene oxide due to increased oxygenated reactive sites delivering enhanced affinity for a wide range of protein biomarkers. OECT devices were also demonstrated for label-free DNA detection, ${ }^{[86]}$ where the device was fabricated on a PET substrate and integrated within a PDMS microfluidic channel. The transistor active layer was PEDOT:PSS with Au electrodes. The device electrical characteristics $\left(I_{\mathrm{DS}}, V_{\mathrm{G}}\right)$ showed similar values before and during bending. The electrical transfer curve shifted positively after DNA hybridization and demonstrated sensitivity down to 10 pM target oligonucleotide with enhanced hybridization conditions. While multiplex target detection was not demonstrated, the potential microarray application could be realized.

\section{Cell targets}

Label-free electrochemical biomolecule detection has generated significant interest in recent years; removing reporter molecules can significantly reduce assay cost and complexity. OECTs have been demonstrated for the detection of sialic acid screening for cancer cells. ${ }^{[87]}$ Such approaches exploit the concept of field-effective transistors; detection is realized by monitoring a source to drain electrical characteristics as gate voltage is modified with biomolecule interaction. In a highly efficient approach for cancer cell detection, sialic acid was directly monitored at a p-aminobenzoic acid-modified gate counter electrode by exploiting direct binding between sialic acid (SA) and phenylboronic acid. The transistor active layer was based on PEDOT:PSS with screen-printed carbon source and drain electrodes. The OECT device showed a response to free SA test solution and HeLa cells with relative standard deviation of $6.3 \%$ (SA) and $12.9 \%$ (HeLa), respectively. The device has demonstrated a potential to distinguish between different cell types (Fig. 6).

\section{Fabric substrates}

Textile-based sensors are a growing research area, most clothing materials are hydrophilic, biocompatible, and naturally conform to the body profile. Approaches to achieve woven fabric sensors include (i) modified fibers/threads, defining conductive paths in cloth or (ii) electrodes printed onto finished garments using flexible print/deposition approaches. Unlike paper and polymer substrates requiring multiple detection and fluidhandling layers, fibers are selectively woven into the fabric, defining sensor and fluidic functionality in a single-layer manufacture process. Textile manufacture incorporates a range of low-cost high-throughput techniques ${ }^{[88]}$ (embroidery, weaving, braiding, coating, and printing) compatible with multiplex biomarker screening devices. Fabrics incorporating measurement technologies are referred to as smart fabric sensors (SFSs). ${ }^{[88]}$
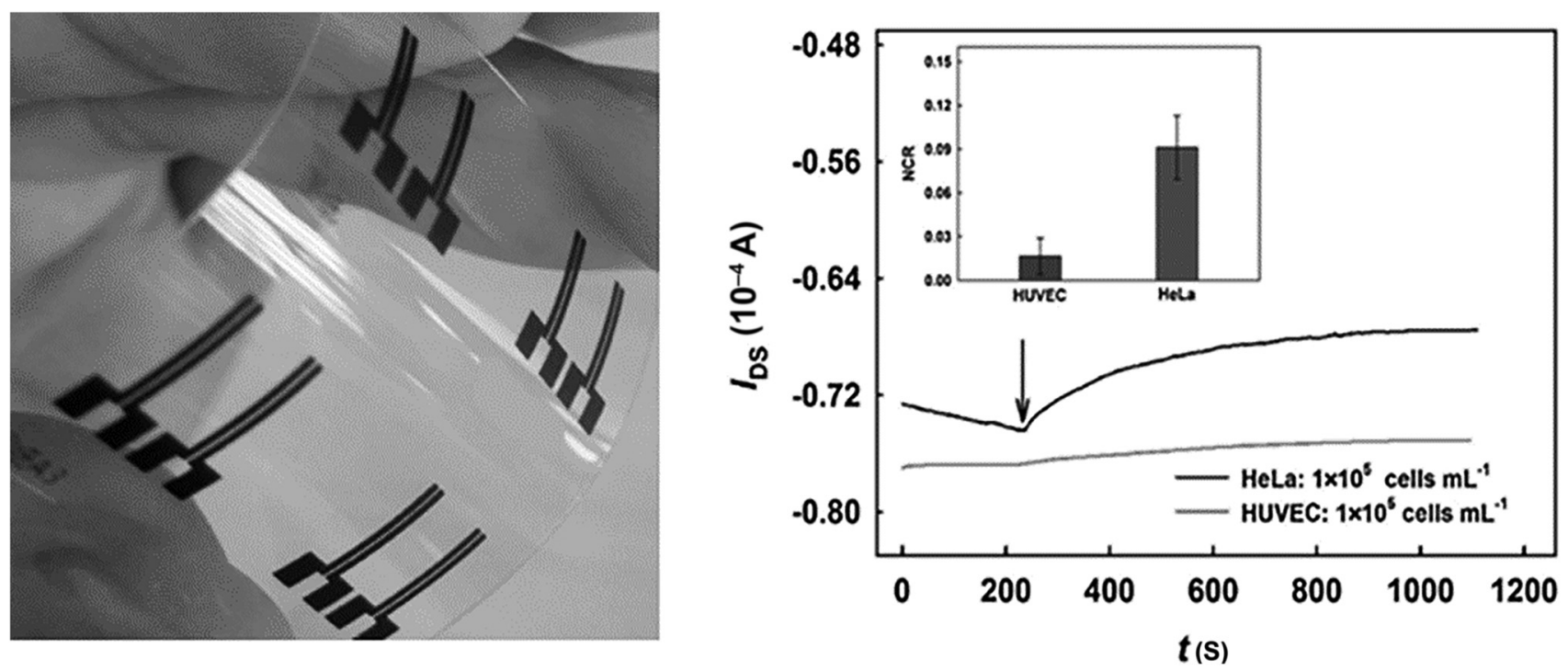

Figure 6. OECT devices were incorporated into a flexible transparent substrate (left) to screen for sialic acid. The potential to differentiate between different cancer (HeLa) and normal (HUVEC) cell types was demonstrated (right) (reprinted from Ref. 87 Copyright Sensors \& Actuators B). 
Stretchable SFSs can be realized by using elastic fibers/ threads, ${ }^{[89]}$ greatly enhancing suitability for wearable applications.

\section{Electrolytes, $\mathbf{p H}$}

Fabric sensors are especially suited to skin applications monitoring sweat or interstitial fluid parameters (e.g., electrolytes). Commercial cotton yarn, dyed with carbon nanotube ink, defined an ion-selective membrane for target-specific measurement. ${ }^{[90]}$ The electrodes were woven into a band aid-type material (Fig. 7), with a commercial miniature RE incorporated into the structure measuring $\mathrm{pH}$, potassium, and nitrates in liquid sample. Optimizing carbon nanotube ink concentrations achieved stable electrode dye for cotton-based applications.

Impedance measurements were made using these band aid devices and yarn-based electrodes were found to be stable over extended time (1 month), with structure bending/stretching having minimal impact on performance. Selectivity and sensitivity of the yarn electrodes were similar to conventional planar electrodes. The device incorporated a cellulose interface between electrodes, which rapidly soaked sweat, eliminating direct skin contact. Device manufacture was convenient and suitable for low-resource countries, due its low manufacture cost and disposable nature. Silk is also an interesting material for fabric-based electrochemical sensors; individual threads were coated with conductive inks and woven into flexible fabric electrodes. ${ }^{[91]}$ Silk threads were coated with reagents and electrode materials before being incorporated into fabric patches realizing sensor arrays. This approach was preferred over conventional screen printing to reduce reagent waste, while incorporating hydrophilic and hydrophobic threads into the sensor design controlled fluid flow and miniaturized sensor footprint.
An OECT formulated on a cotton fiber ${ }^{[92]}$ simultaneously measured adrenaline and $\mathrm{NaCl}$ in sweat sample by oxidation at the gate electrode, forming adrenochrome. Metal wire was used as gate electrodes controlling charge on a PEDOT:PSS conduction channel formulated on a cotton fiber. When switched on, cations flowed from the electrolyte solution to the conductive channel reducing current flow between drain and source; the current amplitude was proportional to sweat adrenaline concentration. The sensor response was measured over the range from $10 \mathrm{nM}$ to $10 \mathrm{mM}$, changing the gate electrode to silverfacilitated salt content measurement.

\section{Glucose, lactate, hemoglobin}

Electrochemical detection was used to detect glucose (chrono-amperometry) and hemoglobin (digital pulse voltammetry). ${ }^{[91]}$ This approach incorporated different reagents on electrodes, in a fashion not previously demonstrated with textile devices, also enhancing mechanical strength for incorporation into wearable devices. The glucose sensor consisted of carbon ink coated onto silk fiber (CE), with thread coated in a carbon ink/potassium ferricyanide mix for the WE, followed by $\mathrm{GOx}$ deposition. An $\mathrm{Ag} / \mathrm{AgCl}$ thread coating was used for the RE. Identical materials and manufacture technique was used for the hemoglobin sensor, with a carbon RE replacing $\mathrm{Ag} / \mathrm{AgCl}$ and WEs left uncoated. For multiplex detection, uncoated carbon REs were incorporated into a four-electrode design with common REs and CEs for multiplex detection. Each sensor was $2 \mathrm{~cm} \times 1.2 \mathrm{~cm} \times 0.1 \mathrm{~cm}$. The multiplex sensor was evaluated with lysed blood sample of known glucose and hemoglobin content. For glucose detection, $5 \mu \mathrm{l}$ of blood was delivered to the sensor and a $0.5 \mathrm{~V}$ fixed voltage was applied between WE and RE with current measured over time.

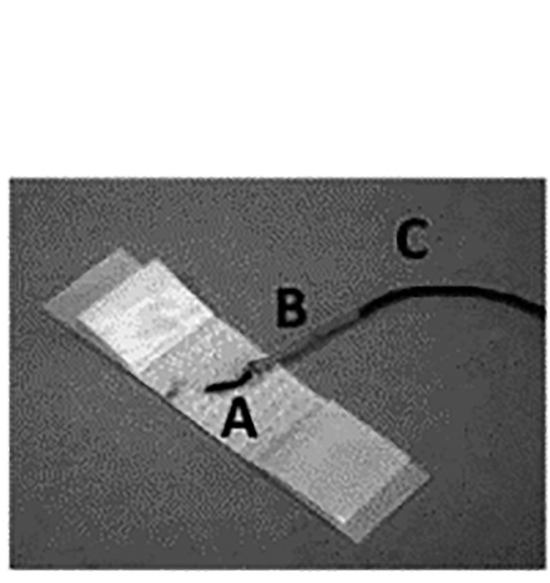

(a)
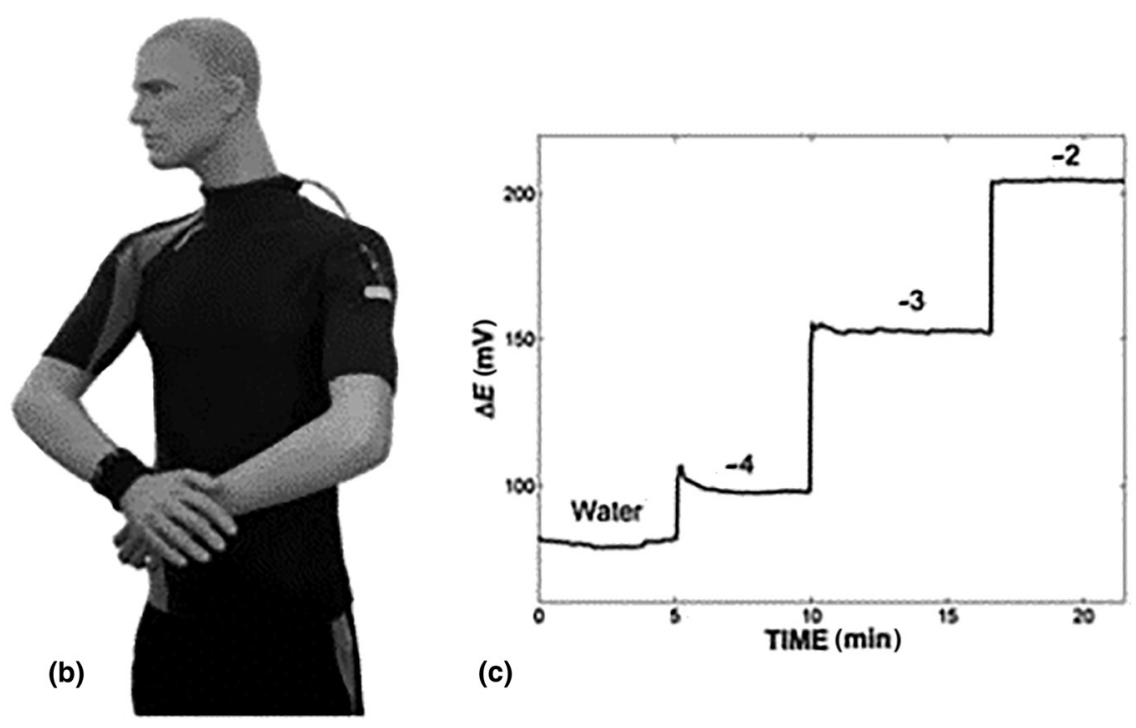

(c)

Figure 7. Band aid plaster material incorporating ion-selective membranes, designed to measure pH, potassium, and nitrates (reprinted from Ref. 90 with permission from Royal Society of Chemistry). 
Glucose was oxidized forming gluconic acid, while potassium ferricyanide reduction produced the measured current. The sensors showed good sensitivity over a clinically relevant range $(80-600 \mathrm{mg} / \mathrm{dL})$ with $<5 \% \mathrm{CV}$. For hemoglobin detection, red blood cell lysis released target into solution. Carbon WEs were used to detect oxyhemoglobin by reduction at $-0.42 \mathrm{~V}$. Using digital peak voltammetry, peak size at $-0.42 \mathrm{~V}$ increased with increasing oxyhemoglobin over the clinically relevant range $2.3-14 \mathrm{~g} / \mathrm{dL}$. Electrochemical sensors were embroidered into fabric, to monitor glucose and lactate in whole blood. ${ }^{[93]}$ Conductive thread was woven to define complex electrode designs using a commercial embroidery machine. Electrochemical detection using complex electrode design patterns was not previously demonstrated with weaved fabric. Carbon ink-coated thread defined the working and $\mathrm{CEs}$, while $\mathrm{Ag} / \mathrm{AgCl}$ ink was used for the RE. Passive enzyme adsorption (e.g., GOx or LOx) was undertaken on the WE for selective detection. Resistivity of the $\mathrm{Ag} / \mathrm{AgCl}$ and carboncoated threads were in line with the literature; to reduce oxidation resistance, the $\mathrm{Ag} / \mathrm{AgCl}$ was flux-coated. The assays were stable, reproducible, and target-specific in liquids containing glucose, lactate, and uric acid. Blood spiked with glucose and lactate was measured over the range $0-40 \mathrm{mM}$, demonstrating suitability for clinical measurements. The sensor performance was stable against mechanical deformation, e.g., bending, stretching, and twisting. An array of graphene FET devices (Fig. 8) suitable for multiplex detection was fabricated on a silk substrate ${ }^{[94]} \mathrm{A}$ thin silk film substrate $(10 \mu \mathrm{m})$ was prepared from silk fibers. GOx was incorporated into silk solutions prior to drying achieving GOx (1\%) loaded substrates. Graphene grown on a nicol (Ni) substrate was transferred to pure silk films using a PDMS stamp technique. Gold electrodes were deposited by low-temperature evaporation $\left(40^{\circ} \mathrm{C}\right)$ and patterned to realize source and drain electrodes on graphene channels. The GOx silk substrate was placed on the silk-graphene layer and adhered using water. The FET gate was then

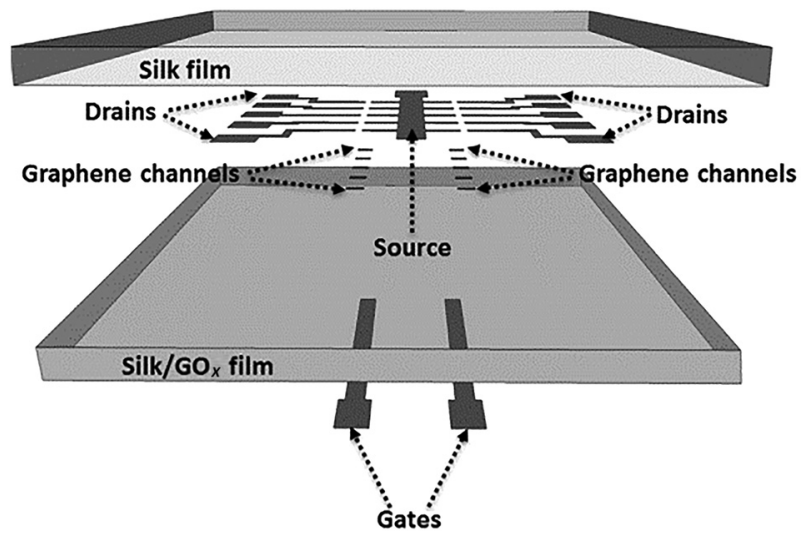

Figure 8. Outline of the incorporation graphene FET device array onto a silk substrate for spatial multiplex detection of glucose in a wearable system (reprinted from Ref. 94 Copyright Sensors \& Actuators B). deposited and patterned on the silk-GOx layer. The glucoseGOx reactions modulated FET conductance, resulting in increased source drain current with increased glucose concentration. With $V_{\mathrm{g}}=0 \mathrm{~V}$ and $V_{\mathrm{ds}}=100 \mathrm{mV}$, the measured current $\left(I_{\mathrm{ds}}\right)$ demonstrated a linear response over glucose concentration $0.1-10 \mathrm{mM}(\mathrm{LOD}=0.1 \mathrm{mM})$, with average sensor sensitivity $2.5 \mathrm{~A} / \mathrm{mM}$. Sensor response time was $<10 \mathrm{~s}$, with up to ten sensors deposited per sensor patch, facilitating multiplex detection. The device was selective with minimal impact from uric acid $(10 \mathrm{mM})$, bovine serum albumin (BSA), or ascorbic acid (10 $\mathrm{mM})$.

Flexible fabric sensors have been extensively deployed for electrophysiologic ${ }^{[95]}$ and blood pressure ${ }^{[96]}$ measurements, and a recent review ${ }^{[97]}$ on flexible strain sensors highlighted polymers and functional nanomaterials emerging in resistive/ capacitive devices. They also highlighted how semiconductor materials significantly improve piezoresistivity over metals in flexible sensor applications; a similar trend is evident with flexible biomarker devices. However, for biomarker monitoring, additional challenges exist around surface fouling, sweat, and humidity. Sampling-appropriate volumes of target biomolecule is also a challenge in wearable devices and may require fluidic reservoir storage or pre-concentration prior to detection.

OECTs were fabricated by coating nylon fibers with multilayers of $\mathrm{Cr} / \mathrm{Au} / \mathrm{PEDOT}: \mathrm{PSS}^{\left[{ }^{[98]}\right.}$ The stretchable fibers were incorporated into the fabric using a commercial weaving system, without any impact on performance. Bending the fiber had a small change in resistance $(26-51 \Omega / \mathrm{cm})$ compared with a fiber coated only with $\mathrm{Cr} / \mathrm{Au}(39-401 \Omega / \mathrm{cm})$. To define the gate electrode, Pt was deposited on the nylon fiber using a Ti adhesion layer. Detection of glucose $\left(30-100 \times 10^{-9} \mathrm{M}\right)$, uric acid, and dopamine was demonstrated with the device. For glucose detection, GOx was deposited on the gate electrode with a modified blocking polymer layer. For UA detection, the gate electrode was modified with Nafion-graphine/PANI/uricase-GO multilayers and detection limits were similar to a planar device $\left(30 \times 10^{-9} \mathrm{M}\right)$ with a linear response up to $300 \times$ $10^{-6} \mathrm{M}$. The gate was modified with graphene flakes for dopamine detection with an LOD $10 \times 10^{-9} \mathrm{M}$. To demonstrate the practical application, the OECT sensors were woven into diapers and used to determine glucose levels in spiked artificial urine.

\section{Concluding remarks and future perspectives}

In recent years, significant growth has occurred with consumer devices, monitoring physical activity (hearth rate, motion, etc.) for the fitness and well-being markets. Flexible substrate sensors based on piezoresistance, piezocapacitance, and piezoelectric technologies have been commercialized where product design delivers robust, easy to use, and comfortable devices. To date, less progress has been made in commercialization of wearable biomarker monitoring devices (e.g., glucose, $\mathrm{Na}^{+}$, etc.). Research and commercial devices have extensively demonstrated single-target biomarker detection; however, there is 
an increased activity around multiplex detection due to more representative diagnostics. New biomarkers emerging from genomic/proteomic research offers opportunities for real-time health monitoring in accessible biofluids (e.g., saliva, sweat, tear fluid). Flexible substrates facilitate on body wearable diagnostics enhancing non-invasive health monitoring. Materials progress has advanced wearable sensors through: (i) biocompatible polymers realizing flexible substrates for integrated electrodes/electronics, (ii) conductive nanomaterials defining flexible electrodes, (iii) target-selective interface layers (ionogels, hydrogels) addressing biofouling and enhancing biomarker selectivity. Integration of graphene, semiconductor, and nanowire materials with conductive polymers has delivered a significant progress in flexible sensor technology by enhancing electrical and mechanical performance. Microfluidics incorporated into flexible sensor devices improve liquid volume sampling ${ }^{[61]}$ and deliver active device metering/ valving. ${ }^{[58]}$

Challenges still exist, e.g., (i) analyte leaching from interface layers, (ii) long-term stability for bio-recognition molecules, and (iii) stable interface potentials between sample and sensor interfaces. Enzyme-free glucose detection has emerged as a key research topic, enabled by enhanced electro-catalytic nanostructure properties. ${ }^{[99,100]}$ These remove surface immobilization and ameliorate issues around enzyme stability and lifetime. However, device reproducibility is an issue with up to $50 \%$ signal variation reported across non-enzyme nanoparticle sensors. ${ }^{[101]}$ Non-linear response/sensitivity and interference from electroactive species is also reported. ${ }^{[101]}$ Enzyme-free electrochemical glucose detection has yet to impact flexible biomarker systems, and enzyme-mediated detection is still widely implemented. For future wearable flexible biomarker monitoring system, sampling will present challenges: (i) minimum sample volumes required to reliably detect target concentrations and (ii) sample/biomarker replenishment rates. Current wearable electrochemical devices have to overcome lifetime and stability issues. Biofouling and electrode poisoning $\left(\mathrm{H}_{2} \mathrm{O}_{2}\right)$ impact long-term stability limiting measurements to $<24 \mathrm{~h}$, acceptable for short-term disposable devices (e.g., contact lens for glucose monitoring) but limiting long-term biomarker monitoring (e.g., days). A challenge for wearable potentiometric devices is avoiding interface equilibrium between RE material and the sample, resulting in a signal drift and limited sensor lifetime. ${ }^{[102]}$ Flexible substrate multiplex biomarker systems are a natural fit for wearable healthmonitoring applications. They can provide rapid, reliable information for the end user, facilitating decision making on medication, performance, or lifestyle. In future developments, flexible polymers with physical characteristics (Young's modulus) similar to skin will advance comfort and robustness of skin-worn devices. For non-invasive monitoring, reliability and clinical performance ${ }^{[103]}$ are key aspects for device performance. Blood diagnostics ${ }^{[104-106]}$ are the benchmark against which wearable systems are evaluated, and lower target concentration, lag times, and sample variation are challenges to be addressed. Progress in wearable sensor technology has been significant; flexible substrates have been facilitated by new nanomaterials, polymers, and novel fabrication technologies. To date, diabetes monitoring has been the commercial and technology benchmark, ${ }^{[107-109]}$ with glucose and lactate extensively used to demonstrate proof of concept in research devices. ${ }^{[110-115]}$ Emerging biomarkers from genome and proteome research are generating clinical interest as biomarkers linked to specific diseases. Currently, many commercial diagnostic assays monitor single biomarkers (e.g., glucose), but the possibility to monitor multiple biomarkers promises more efficient health monitoring where multiple parameters inform clinical diagnosis. The potential for non-invasive wearable biomedical sensors as alternatives to invasive monitoring is significant and the explosion in physical activity and lifestyle monitoring devices illustrate how issues around biofouling, calibration, and signal interference can be addressed.

\section{Supplementary material}

The supplementary material for this article can be found at https://oi.org/10.1557/mrc.2018.134.

\section{Acknowledgments}

CALIN-Ireland Wales InterReg Programme. SFI Insight Centre.

\section{References}

1. W. Yan, R. Apweiler, B.M. Balgley, P. Boontheung, J.L. Bundy, B. J. Cargile, S. Cole, X. Fang, M. Gonzalez-Begne, T.J. Griffin, F. Hagen, S. Hu, L.E. Wolinsky, C.S. Lee, D. Malamud, J.E. Melvin, R. Menon, M. Mueller, R. Qiao, N.L. Rhodus, J.R. Sevinsky, D. States, J. L. Stephenson, S. Than, J.R. Yates, W. Yu, X. Xie, Y. Xie, G. S. Omenn, J.A. Loo, and D.T. Wong: Systematic comparison of the human saliva and plasma proteomes. Proteomics Clin. Appl. 3, 116134 (2009).

2. M. Soukup, I. Biesiada, A. Henderson, B. Idowu, D. Rodeback, L. Ridpath, E.G. Bridges, A.M. Nazar, and K.G. Bridges: Salivary uric acid as a noninvasive biomarker of metabolic syndrome. Diabetol. Metab. Syndr. 4, 14 (2012).

3. R. Semba, J. Enghild, V. Venkatraman, T. Dyrlund, and J.E. Van Eyk: The human eye proteome project: perspectives on an emerging proteome. Proteomics 13, 2500-2511 (2013).

4. C. Huang, M. Chen, L. Huang, and I. Mao: Uric acid and urea in human sweat. Chin. J. Physiol. 45, 109-115 (2002).

5. W.A. Latzka and S.J. Montain: Water and electrolyte requirements for exercise. Clin. Sports Med. 18, 513-524 (1999).

6. S. Shirreffs, L. Aragon-Vargas, M. Chamorro, R. Maughan, L. Serratosa, and J. Zachwieja: The sweating response of elite professional soccer players to training in the heat. Int. J. Sports Med. 26, 90-95 (2005).

7. W. Zeng, L. Shu, Q. Li, S. Chen, F. Wang, and X. Tao: Fiber based wearable electronics: a review of materials, fabrication, devices, and applications. Adv. Mater. 26, 5310-5336 (2014).

8. S. Choi, H. Lee, R. Ghaffari, T. Hyeon, and D.H. Kim: Recent advances in flexible and stretchable bio-electronic devices integrated with nanomaterials. Adv. Mater. 28, 4203-4218 (2016).

9. M. Stoppa and A. Chiolerio: Wearable electronics and smart textiles: a critical review. Sensors 14, 11957-11992 (2014).

10. M. Raiszadeh, M. Ross, and P. Russo: Proteomic analysis of eccrine sweat: implications for the discovery of schizophrenia biomarker proteins. J. Proteome Res. 11, 2127-2139 (2012).

11. S. Park, J. Ahn, X. Feng, S. Wang, Y. Huang, and J.A. Rogers: Theoretical and experimental studies of bending of inorganic electronic 
materials on plastic substrates. Adv. Funct. Mater. 18, 2673-2684 (2008).

12. F.H. Silver, J.W. Freeman, and D. DeVore: Viscoelastic properties of human skin and processed dermis. Skin Res. Technol. 7, 18-23 (2001).

13. L. Peng, Z. Dongzhi, L. Jingjing, C. Hongyan, S. Yan, and Y. Nailiang: Air-stable black phosphorus devices for ion sensing. ACS Appl. Mater. Interfaces 7, 24396-24402 (2015).

14. Y. Bo, H. Yang, Y. Hu, T. Yao, and S. Huang: A novel electrochemical DNA biosensor based on graphene and polyaniline nanowires. Electrochim. Acta 56, 2676-2681 (2011).

15. X. Kang, J. Wang, H. Wu, I.A. Aksay, J. Liu, and Y. Lin: Glucose oxidasegraphene-chitosan modified electrode for direct electrochemistry and glucose sensing. Biosens Bioelectron. 25, 901-905 (2009).

16. S. Kumar Vashist, D. Zheng, K. Al-Rubeaan, J.H.T. Luong, and F. S. Sheu: Advances in carbon nanotube based electrochemical sensors for bioanalytical applications. Biotechnol. Adv. 29, 169-188 (2011).

17. C.B. Jacobs, M.J. Peairs, and B.J. Venton: Review: carbon nanotube based electrochemical sensors for biomolecules. Anal. Chim. Acta 662, 105-127 (2010).

18. K. Saha, S.S. Agasti, C. Kim, X. Li, and V.M. Rotello: Gold nanoparticles in chemical and biological sensing. Chem. Rev. 112, 27392779 (2012).

19. X. Luo, A. Morrin, A.J. Killard, and M.R. Smyth: Application of nanoparticles in electrochemical sensors and biosensors. Electroanalysis 18, 319-326 (2006).

20. S.R. Forrest: The path to ubiquitous and low-cost organic electronic appliances on plastic. Nature 428, 911-918 (2004).

21. Y. Liu, L. Shi, M. Wang, Z. Li, H. Liu, and J. Shilpa: A novel room temperature ionic liquid sol-gel matrix for amperometric biosensor application. Green Chem. 7, 655-658 (2005).

22. N. Nishi, H. Murakami, Y. Yasui, and T. Kakiuchi: Use of highly hydrophobic ionic liquids for ion-selective electrodes of the liquid membrane type. Anal. Sci. 24, 1315-1320 (2008).

23. B. Penga, J. Zhub, X. Liua, and Y. Qina: Potentiometric response of ionselective membranes with ionic liquids as ion-exchanger and plasticizer. Sens. Actuators B 133, 308-314 (2008).

24. G. Reach: Continuous glucose monitoring and diabetes health outcomes: a critical appraisal. Diab. Technol. Ther. 10, 69-80 (2008).

25. S. Iguchi and H.T. Saito: A flexible and wearable biosensor for tear glucose measurement. Biomed. Microdevices 9, 603 (2007).

26. M.F. Bergeron: Heat cramps: fluid and electrolyte challenges during tennis in the heat. J. Sci. Med. Sport 6, 19-27 (2003).

27. L.S. Selva-Kumar, X. Wang, J. Hagen, R. Naik, I. Papautsky, and J. Heikenfeld: Label free nano-aptasensor for interleukin-6 in protein-dilute biofluids such as sweat. Anal. Methods 8, 3440-3444 (2016).

28. C.G. Fraga: Relavance, essentiality and toxicity of trace elements in human health. Mol. Aspects Med. 26, 235 (2005).

29. S.R. Newmark and R.G. Dluhy: Hyperkalemia and hypokalemia. JAMA 231, 631-633 (1975)

30. M. Gamella: A novel non-invasive electrochemical biosensing device for in situ determination of the alcohol content in blood by monitoring ethanol in sweat. Anal. Chim. Acta 806, 1-7 (2014).

31. T. Umeda: Use of saliva for monitoring unbound free cortisol levels in serum. Clin. Chim. Acta 110, 245-253 (1981).

32. P. Zuo, X. Li, D. Dominguez, and B. Yeb: A PDMS/paper/glass hybrid microfluidic biochip integrated with aptamer-functionalized graphene oxide nano-biosensors for one-step multiplexed pathogen detection. Lab Chip 13, 3921-3928 (2013).

33. M. Sher, R. Zhuang, U. Demirci, and W. Asghar: Paper-based analytical devices for clinical diagnosis: recent advances in the fabrication techniques and sensing mechanisms. Expert Rev. Mol. Diagn. 17, 351366 (2017).

34. J.P. Comer: Semi quantitative specific test paper for glucose in urine. Anal. Chem. 28, 1748-1750 (1956).

35. A.W. Martinez, S.T. Phillips, M.J. Butte, and G.M. Whitesides: Patterned paper as a platform for inexpensive, low-volume, portable bioassays. Angew. Chem. Int. Ed. 46, 1318-1320 (2007).
36. A.W. Martinez, S.T. Phillips, B.J. Wiley, M. Gupta, and G.M. Whitesides: Flash: a rapid method for prototyping paper-based microfluidic devices. Lab. Chip 8, 2146-2150 (2008).

37. R. Mukhopadhyay: Cheap, handheld colorimeter to read paper-based diagnostic devices. Anal. Chem. 81, 8659 (2009).

38. A.W. Martinez, S.T. Phillips, and G.M. Whitesides: Diagnostics for the developing world: microfluidic paper-based analytical devices. Anal. Chem. 82, 3-10 (2010).

39. S.K. Sia and L.J. Kricka: Lab on paper. Lab. Chip 8, 1988-1991 (2008)

40. G.M. Whitesides: What comes next? Lab. Chip 11, 191-193 (2011).

41. R. Mukhopadhyay: Medical diagnostics with paper and camera phones. Anal. Chem. 80, 3949 (2008).

42. Y. Lu, W. Shi, L. Jiang, J. Qin, and B. Lin: Rapid prototyping of paperbased microfluidics with wax for low-cost, portable bioassay. Electrophoresis 30, 1497-1500 (2009).

43. S.K. Tang and G.M. Whitesides: Optofluid.: Fundam. Devices Appl. 1, 731 (2010)

44. W. Dungchai, O. Chailapakul, and C.S. Henry: Electrochemical detection for paper-based microfluidics. Anal. Chem. 81, 5821-5826 (2009).

45. A.W. Martinez, S.T. Phillips, E. Carrilho, S.W. Thomas, H. Sindi, and G. M. Whitesides: Simple telemedicine for developing regions: camera phones and paper-based microfluidic devices for real-time, off-site diagnosis. Anal. Chem. 80, 3699-3707 (2008).

46. F. Kivlehan, M. Paolucci, D. Brennan, I. Ragoussis, and P. Galvin: Threedimensional hydrogel structures as optical sensor arrays, for the detection of specific DNA sequences. Anal. Biochem. 421, 1-8 (2012).

47. Y. Yao and C. Zhang: A novel screen-printed microfluidic paper-based electrochemical device for detection of glucose and uric acid in urine. Biomed. Microdevices 18, 92 (2016).

48. X. Wei, T. Tian, S. Jia, Z. Zhu, Y. Ma, J. Sun, Z. Lin, and C. Yang: Microfluidic distance readout sweet hydrogel integrated paper. Based analytical device ( $\mu$ DiSH-PAD) for visual quantitative point of-care testing. Anal. Chem. 88, 2345-2352 (2016).

49. Y.F. Zheng, G.W. Xu, D.Y. Liu, J.H. Xiong, P.D. Zhang, C. Zhang, Q. Yang, and S. Lv: Study of urinary nucleosides as biological marker in cancer patients analyzed by micellar electrokinetic capillary chromatography. Electrophoresis 23, 4104-4109 (2002).

50. S. Wang, L. Ge, X. Song, J. Yu, S. Ge, J. Huang, and F. Zeng: Paperbased chemiluminescence ELISA: Lab-on-paper based on chitosan modified paper device and wax-screen-printing. Biosens. Bioelectron. 31, 212-218 (2012).

51. L. Ge, J. Yan, X. Song, M. Yan, S. Ge, and J. Yu: Three-dimensional paper-based electrochemiluminescence immunodevice for multiplexed measurement of biomarkers and point-of-care testing. Biomaterials 33, 1024-1031 (2012).

52. L. Wu, C. Ma, L. Ge, Q. Kong, M. Yan, S. Ge, and J. Yu: Paper-based electrochemiluminescence origami cyto-device for multiple cancer cells detection using porous AuPd alloy as catalytically promoted nanolabels. Biosens. Bioelectron. 63, 450-457 (2015).

53. M. Su, L. Ge, S. Ge, N. Li, J. Yu, M. Yan, and J. Huang: Paper based electrochemical cyto device for sensitive detection of cancer cells. Anal. Chim. Acta 847, 1-9 (2014).

54. M. Su, L. Ge, Q. Kong, X. Zheng, S. Ge, N. Li, J. Yu, and M. Yan: Polymer based devices. Biosens. Bioelectron. 63, 232-239 (2015).

55. M. Yang, W Zhang, J. Yang, B. Hu, F. Cao, W. Zheng, Y. Chen, and $X$. Jiang: Skiving stacked sheets of paper into test paper for rapid and multiplexed assay. Sci. Adv. 3, eaa04862 (2017).

56. J. Cunningham, N. Brenes, and R. Crooks: Paper electrochemical device for detection of DNA and thrombin by target-induced conformational switching. Anal. Chem. 86, 6166-6170 (2014).

57. P. Ihalainen, F. Pettersson, M. Pesonen, T. Viitala, A. Määttänen, R. Österbacka, and J. Peltonen: An impedimetric study of DNA hybridization on paper-supported inkjet-printed gold electrodes. Nanotechnology 25, 094009 (2014) (11pp).

58. J. Wang, W. Li, L. Ban, W. Du, X. Feng, and B. Liu: A paper-based device with an adjustable time controller for the rapid determination of tumour biomarkers. Sens. Actuators B 254, 855-862 (2018).

59. W. Gao, S. Emaminejad, H. Nyein, S. Challa, K. Chen, A. Peck, H. Fahad, H. Ota, H. Shiraki, D. Kiriya, D. Lien, G. Brooks, R. Davis, and A. Javey: 
Fully integrated wearable sensor arrays for multiplexed in situ perspiration analysis. Nature 529, 509-514 (2016).

60. S. Emaminejada, W. Gao, E. Wub, Z.A. Davies, H. Nyein, S. Challaa, S. P. Ryan, H. Fahad, K. Chen, Z. Shahpar, S. Talebia, C. Millaf, A. Javey, and R.W. Davies: Autonomous sweat extraction and analysis applied to cystic fibrosis and glucose monitoring using a fully integrated wearable platform. Proc. Natl. Acad. Sci. 114, 4625-4360 (2017).

61. A. Martín, J. Kim, J.F. Kurniawan, J.R. Sempionatto, J.R. Moreto, G. Tang, A.S. Campbell, A. Shin, M. Lee, X. Liu, and J. Wang: Epidermal microfluidic electrochemical detection system: sweat sampling and metabolite detection. ACS Sens. 2, 1860-1868 (2017).

62. M. Parrilla, J. Ferr, T. Guinovart, and F. Andrade: Wearable potentiometric sensors based on commercial carbon fibres for monitoring sodium in sweat. Electroanalysis 28, 1267-1275 (2016).

63. G. Matzeu, C.O. Quigley, E. McNamara, C. Zuliani, C. Fay, T. Glennon, and D. Diamond: An integrated sensing and wireless communications platform for sensing sodium in sweat. Anal. Methods 8, 64-71 (2016).

64. Y. Rim, S. Bae, H. Chen, J.L. Yang, J. Kim, A.M. Andrews, P.S. Weiss, Y. Yang, and $\mathrm{H}$. Tseng: Printable ultrathin metal oxide semiconductorbased conformal biosensors. ACS Nano 9, 12174-12181 (2015).

65. C. Liao, C. Mak, M. Zhang, H.W. Chan, and F. Yan: Flexible organic electrochemical transistors for highly selective enzyme biosensors and used for saliva testing. Adv. Mater. 27, 676-681 (2015).

66. S. Anastasova, B. Crewther, P. Bembnowicz, V. Curto, H. Ip, B. Rosa, and G.-Z. Yang: A wearable multisensing patch for continuous sweat sensing. Biosens. Bioelectron. 93, 139-145 (2017).

67. Y. Sekine, S. Kim, Y. Zhang, A. Bandodkar, S. Xu, J. Choi, M. Irie, T. Ray, P. Kohli, N. Kozai, T. Sugita, Y. Wu, K. Lee, K. Lee, R. Ghaffarid, and J. A. Rogers: A fluorometric skin-interfaced microfluidic device and smartphone imaging module for in situ quantitative analysis of sweat chemistry. Lab. Chip (2018). Advance Article on June 29, DOI: 10.1039/ C8LC00530C

68. A. Bhide, S. Muthukumar, A. Saini, and S. Prasad: Simultaneous lancetfree monitoring of alcohol and glucose from low-volumes of perspired human sweat. Sci. Rep. 8, 6507 (2018).

69. S. Imani, A. Bandodkar, A. Vinu Mohan, R. Kumar, S. Yu, J. Wang, and P. Mercier: A wearable chemical-electrophysiological hybrid biosensing system for realtime health and fitness monitoring. Nat. Commun. 7, 11650 (2016).

70. B. Bansoda, T. Kumarb, R. Thakurc, S. Ranac, and I. Singh: A review on various electrochemical techniques for heavy metal ions detection with different sensing platforms. Biosens. Bioelectron. 94, 443-455 (2017).

71. G. March, T. Nguyen, and B. Piro: Modified electrodes used for electrochemical detection of metal ions in environmental analysis. Biosensors 5. 241-275 (2015).

72. G. Zhao, Y. Sheng, H. Wang, and G. Liu: A portable electrochemical detection system based on graphene/ionic liquid modified screenprinted electrode for the detection of cadmium in soil by square wave anodic stripping voltammetry. Int. J. Electrochem. Sci. 11, 54-64 (2016).

73. W. Yantasee, Y. Lin, K. Hongsirikarn, G.E. Fryxell, R. Addleman, and C. Timchalk: Electrochemical sensors for the detection of lead and other toxic heavy metals: the next generation of personal exposure biomonitors. Environ. Health Perspect. 115, 1683-1690 (2007).

74. X. Xuan, F. Hossain, and J. Park: A fully integrated and miniaturized heavy-metal-detection sensor based on micro-patterned reduced graphene oxide. Sci. Rep. 6, 33125 (2016).

75. M. Schaefer, M. Schellenberg, U. Merle, K.H. Weiss, and W. Wilson: Protein expression, copper excretion and sweat production in sweat glands of Wilsons disease patients and controls. BMC Gastroenterol. 8, 29-31 (2008).

76. A. Crew, D. Cowell, and J.P. Hart: Development of an anodic stripping voltammetric assay, using a disposable mercury free screen printed carbon electrode for determination of Zinc in human sweat. Talanta 75, 1221-1226 (2008).

77. A.P. De Souza, A.S. Lima, M.O. Salles, A.N. Nascimento, and M. Bertotti: The use of a gold disc microelectrode for the determination of copper in human sweat. Talanta 83, 167-170 (2010).
78. J. Kim, W.R. de Araujo, I.A. Samek, A.J. Bandodkar, W. Jia, B. Brunetti, T. Paixao, and J. Wang: Wearable temporary tattoo sensor for real time trace metal monitoring in human sweat. Electrochem. Commun. 51, 4145 (2015)

79. W. Goa, H.Y. Nyein, Z. Shahpar, H.M. Fahad, K. Chen, S. Emaminejad, Y. Goa, L. Tai, H. Ota, E. Wu, J. Bullock, Y. Zeng, D. Lein, and A. Javey: Heavy metal monitoring of bodily fluids. ACS Sens. 1, 866874 (2016).

80. A. Koh, D. Kang, Y. Xue, S. Lee, R. Pielak, J. Kim, T. Hwang, S. Min, A. Banks, P. Bastien, M. Manco, L Wang, K. Ammann, K. Jang, P. Won, S. Han, R. Ghaffari, U. Paik, M. Slepian, G. Balooch, Y. Huang, and J.A. Rogers: A soft, wearable microfluidic device for the capture, storage, and colorimetric sensing of sweat. Sci. Transl. Med. 8, 165-185 (2016).

81. A. Baraket, M. Lee, N. Zine, M. Sigaud, N. Yaakoubi, M. Trivella, M. Zabala, J. Bausells, N. Jaffrezic-Renault, and A. Errachid: Diazonium modified gold microelectrodes onto polyimide substrates for impedimetric cytokine detection with an integrated $\mathrm{Ag} / \mathrm{AgCl}$ reference electrode. Sens. Actuators B 189, 165-172 (2013).

82. A. Garcia-Cruz, M. Lee, N. Zine, M. Sigaud, J. Bausells, and A. Errachid: Poly(pyrrole) microwires fabrication process on flexible thermoplastic polymers: application as a biosensing material. Sens. Actuators $B$ 221, 940-950 (2015).

83. V. Kamakoti, A. Selvam, N. Shanmugam, S. Muthukum, and S. Prasad: Flexible molybdenum electrodes towards designing affinity based protein biosensors. Biosensors 6, 36 (2016).

84. H. Pei, J. Li, M. Lv, J. Wang, J. Gao, J. Lu, Y. Li, Q. Huang, J. Hu, and C. Fan: A graphene-based sensor array for high-precision and adaptive target identification with ensemble aptamers. J. Am. Chem. Soc. 134, 13843-13849 (2012)

85. S. Chou, M. De, J. Luo, M. Rotello, J. Huang, and V. Dravid: Nanoscale graphene oxide (nGO) as artificial receptors: implications for biomolecular interactions and sensing. J. Am. Chem. Soc. 134, 16725-16733 (2012).

86. P. Lin, X. Luo, I. Hsing, and F. Yan: Organic electrochemical transistors integrated in flexible microfluidic systems and used for label-free DNA sensing. Adv. Mater. 23, 4035-4040 (2011).

87. X. Guo, J. Liu, F. Liu, F. She, Q. Zheng, H. Tang, M. Ma, and S. Yao: Label-free and sensitive sialic acid biosensor based on organic electrochemical transistors. Sens. Actuators B 240, 1075-1082 (2017).

88. L. Castano and A. Flatau: Smart fabric sensors and e-textile technologies: a review. Smart Mater. Struct. 23, 053001 (2014) (pp27).

89. Ohmatex-Smart Textile Technology. Available at www.ohmatex.dk (accessed February 21, 2018).

90. T. Guinovart, M. Parilla, G.A. Crespo, F.X. Rius, and F.J. Andrade: Potentiometric sensors using cotton yarns, carbon nanotubes and polymeric membranes. Analyst 138, 5208-5215 (2013).

91. T. Choudhary, G. Rajamanickam, and D. Dendukuri: Woven electrochemical fabric based test sensors: a new class of multiplexed electrochemical sensors. Lab. Chip 15, 2064-2072 (2015).

92. N. Coppede, G. Tarabella, M. Villani, D. Calestani, S. Lannotta, and A. Zappettini: Human stress monitoring through an organic cotton-fiber biosensor. J. Mater.Chem. B 2, 5620 (2014).

93. X. Liu and P.B. Lillehoj: Embroidered electrochemical sensors for biomolecule detection. Lab. Chip 16, 2093-2098 (2016).

94. X. You and J.J. Pak: Graphene-based field effect transistor enzymatic glucose biosensor using silk protein for enzyme immobilization and device substrate. Sens. Actuators B 202, 1357-1365 (2014).

95. H. Chou, A. Nguyen, A. Chortos, J. To, C. Lu, J. Mei, T. Kurosawa, W. Bae, J. Tok, and Z. Bao: A chameleon inspired stretchable electronic skin with interactive colour changing controlled by tactile sensing. Nat. Commun. 6, 8011 (2015)

96. S. Lee, A. Reuveny, J. Reeder, J. Lee, H. Jin, Q. Liu, T. Yokota, T. Sekitani, T. Isoyama, Y. Abe, Z. Suo, and T. Someya: A transparent bending-insensitive pressure sensor. Nat. Nanotechnol. 11, 472 (2016).

97. M. Amjadi, K. Kyung, I. Park, and M. Sitti: Stretchable, skin-mountable, and wearable strain sensors and their potential applications: a review. Adv. Funct. Mater. 26, 1678-1698 (2016). 
98. A. Yang, Y. Li, C. Yang, Y. Fu, N. Wang, L. Li, and F. Yan: Fabric organic electrochemical transistors for biosensors. Adv. Mater. 30, 1800051 (2018).

99. Q. Baloach, A. Tahira, A. Begum Mallah, M. Ishaq Abro, S. Uddin, M. Willander, and Z. Ibupoto: A robust, enzyme-free glucose sensor based on lysine-assisted CuO nanostructures. Sensors 16, 1878 (2016).

100.Y. Bai, W. Yang, Y. Sun, and C. Sun: Enzyme-free glucose sensor based on a three-dimensional gold film electrode. Sens. Actuators B 134, 471476 (2008).

101.C. Bell, A. Nammari, P. Uttamchandani, A. Rai, P. Shahand, and A. Moore: Flexible electronics-compatible non-enzymatic glucose sensing via transparent CuO nanowire networks on PET films. Nanotechnology 28, 245502 (2017) (11pp).

102.D. Choi, J. Kim, G.R. Cutting, and P. Searson: Wearable potentiometric chloride sweat sensor: the critical role of the salt bridge. Anal. Chem. 88, 12241-12247 (2016).

103.A.P.F. Turner: Biosensors: sense and sensibility. Chem. Soc. Rev. $\mathbf{4 2}$, 3184-3196 (2013).

104.M. de Planell-Saguer and M. Celina Rodicio: Analytical aspects of microRNA in diagnostics: a review. Anal. Chim. Acta 699, 134-152 (2011).

105.S. Dijkstra, P.F.A. Mulders, and J.A. Schalken: Clinical use of novel urine and blood based prostate cancer biomarkers: a review. Clin. Biochem. 47, 889-896 (2014).

106.T. Monbailliu, J. Goossens, and S. Hachimi-Idrissi: Blood protein biomarkers as diagnostic tool for ischemic stroke: a systematic review. Biomark. Med. 11, 503-512 (2017).

107.https://www.medtronic-diabetes.ie/minimed-system/continuous-glucosemonitoring (accessed February 2, 2018).

108. https://freestylediabetes.ie/ (accessed February 2, 2018).

109.http://www.gluco-wise.com/ (accessed February 2, 2018).

110. H. Lee, T. Choi, Y. Lee, H. Cho, R. Ghaffari, L. Wang, H. Choi, T. Chung, N. Lu, T. Hyeon, S. Choi, and D. Kim: A graphene-based electrochemical device with thermoresponsive microneedles for diabetes monitoring and therapy. Nat. Nanotechnol. 11, 566-572 (2016).

111.W. Jia: Electrochemical tattoo biosensors for real-time noninvasive lactate monitoring in human perspiration. Anal. Chem. 85, 6553-6560 (2013).

112.J. Kim, G. Valdés-Ramírez, A.J. Bandodkar, W. Jia, A.G. Martinez, J. Ramírez, P. Mercier, and J. Wang: Non-invasive mouthguard biosensor for continuous salivary monitoring of metabolites. Analyst 139, 1632 (2014).

113.R. Malon, K.Y. Chua, D.H. Wicaksono, and E. Corcoles: Cotton fabric based electrochemical device for lactate measurement in saliva. Analyst 139, 3009 (2014).

114.S. Liakat, K.A. Bors, L. Xu, C.M. Woods, J. Doyle, and C.F. Gmachl: Noninvasive in vivo glucose sensing on human subjects using midinfrared light. Biomed. Opt. Express 5, 2397-2404 (2014).

115.N. Ozana, Y. Beiderman, V. Mico, M. Sanz, X. Garcia, A. Arnand, J. Baharam, Y. Epstein, and Z. Zalevsky: Improved noncontact optical sensor for detection of glucose concentration and indication of dehydration level. Biomed. Opt. Express 5, 1926-1940 (2014). 